\title{
PRINCIPAL 2-BLOCKS OF THE SIMPLE GROUPS OF REE TYPE
}

BY

\author{
PETER LANDROCK ${ }^{1}$ AND GERHARD O. MICHLER $^{2}$
}

\begin{abstract}
ABSTRACr. The decomposition numbers in characteristic 2 of the groups of Ree type are determined, as well as the Loewy and socle series of the indecomposable projective modules. Moreover, we describe the Green correspondents of the simple modules. As an application of this and similar works on other simple groups with an abelian Sylow 2-subgroup, all of which have been classified apart from those considered in the present paper, we show that the Loewy length of an indecomposable projective module in the principal block of any finite group with an abelian Sylow 2-subgroup of order $2^{n}$ is bounded by $\max \left\{2 n+1,2^{n}\right\}$. This bound is the best possible.
\end{abstract}

Introduction. The purpose of this paper is to determine the algebra structure of the principal 2-blocks $B$ of the simple groups $R(q)$ of Ree type of order $|R(q)|=$ $\left(q^{3}+1\right) q^{3}(q-1)$, where $q=3^{2 n+1}$, and $n=1,2, \ldots$

Let $(F, R, S)$ be a splitting 2-modular system for $R(q)$, where $F$ has characteristic 2 , and $S$ and $R$ have characteristic zero. The character table of the groups $R(q)$ was determined by Ward [15] up to a few but very essential values missing because of the incomplete classification of these groups. Ward [15] also showed that $B$ contains eight ordinary irreducible characters $\xi_{i}$, all of height zero, and five nonisomorphic simple $F R(q)$-modules $\varphi_{i}, i=1,2, \ldots, 5$, where $\varphi_{1}=I$ denotes the trivial $F R(q)$-module. In [7], Fong determined the decomposition matrix up to three parameters $a, b$ and $c$. He also showed that we may choose notation so that $\varphi_{2}$ and $\varphi_{3}$ are algebraically invariant and self-dual, while $\varphi_{4}$ and $\varphi_{5}$ are the duals and the algebraic conjugates of each other.

In $\$ \S 2$ and 3 we complete the decomposition matrix of $B$ by showing that $a=2$ and $b=c=1$ for every $n=1,2, \ldots$ (Theorem 3.9).

If $M$ is a finitely generated $F G$-module, $G$ a finite group, then $\operatorname{soc}(M)=S_{1}(M)$ denotes the socle of $M$ which is the sum of all simple $F G$-modules of $M$. Let $S_{i+1}(M) / S_{i}(M)=S_{1}\left(M / S_{i}(M)\right)$. Then $0<S_{1}(M)<S_{2}(M)<\cdots<S_{k-1}(M)$ $<S_{k}(M)=M$ is called the socle series of $M$, and $k$ is the socle length of $M$ which coincides with the Loewy length $j(M)$ of $M$. If $J$ denotes the Jacobson radical of $F G$, then $j(M)$ is the uniquely determined integer $j$ with $M J^{j-1} \neq 0=M J^{j}$. In order to describe the socle series of an $F G$-module $M$, we associate to $M$ a matrix

Received by the editors November 11, 1978 and, in revised form, August 20, 1979.

AMS (MOS) subject classifications (1970). Primary 20C20, 20C30; Secondary 16A64.

Key words and phrases. Decomposition numbers, projective modules, Green correspondents, simple modules, groups of Ree type.

'Supported in part by DAAD, Bonn, and The Danish Natural Science Research Council.

${ }^{2}$ Supported by the Mathematics Institute of Aarhus University. 
which in the $i$ th row vector has the composition factors of $S_{i}(M) / S_{i-1}(M)$ with their multiplicities.

Let $D$ be a Sylow 2-subgroup of $R(q)$. Then $D$ is an elementary abelian group $D$ of order $|D|=8$. Its normalizer $N=N_{R(q)}(D)$ is a holomorph of $D$ by a noncyclic Frobenius group $F_{21}$ of order $\left|F_{21}\right|=21$. Hence $F N$ is a block algebra. Its structure was determined by the authors in [12]. It has five nonisomorphic simple $F N$-modules, $I$ and four others denoted by $1,1^{*}, 3$ and $3^{*}$, where our notation indicates the degree of the simple $F N$-module, and where $m^{*}$ denotes the dual of $m$. By Knörr's theorem [10], each simple $B$-module $\varphi_{i}$ has vertex $\operatorname{vx}\left(\varphi_{i}\right)={ }_{R(q)} D$. Therefore we consider the Green correspondence $f$ with respect to $D$ between the indecomposable $F R(q)$-modules of $B$ and the indecomposable $F N$-modules. The main results of $\$ \S 2$ and 5 are collected in Theorem 5.3 which asserts that the socle series of the Green correspondents $f\left(\varphi_{i}\right)$ are:

(a) $f(I)=I, f\left(\varphi_{4}\right)=1^{*}$, and $f\left(\varphi_{5}\right)=1$,

(b) $f\left(\varphi_{2}\right)=\frac{3^{*}}{3}$

3

(c) $f\left(\varphi_{3}\right)=3 \quad 3^{*} \quad 3^{*}$

These Green correspondents are used to compute the parameters $a$ and $b$ of the decomposition matrix of $B$ in $\$ \S 2$ and 3. Furthermore, they enable us to find the structure of the indecomposable projective $B$-modules $\boldsymbol{P}_{i}$, where $\boldsymbol{P}_{i}$ denotes the projective cover of $\varphi_{i}, i=1,2, \ldots, 5$.

THEOREM 4.1. The indecomposable projective $F R(q)$-modules of the principal 2block $B$ of any simple group $R(q)$ of Ree type have Loewy and socle series:

(a)

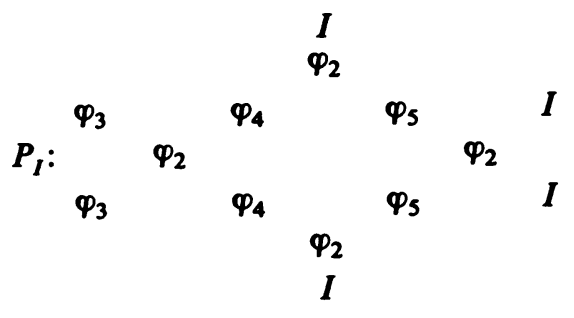

(b)

$\begin{array}{ccccccc}I & & \varphi_{3} & & \varphi_{4} & & \varphi_{5} \\ & \varphi_{2} & & \varphi_{2} & & \varphi_{2} & \\ P_{2} & \varphi_{5} & \varphi_{4} & \varphi_{3} & \varphi_{4} & \varphi_{5} & I \\ & \varphi_{2} & & \varphi_{2} & & \varphi_{2} & \\ I & & \varphi_{3} & & \varphi_{4} & & \varphi_{5}\end{array}$


(c)

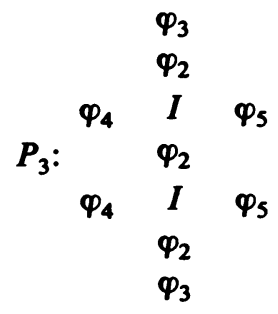

(d)

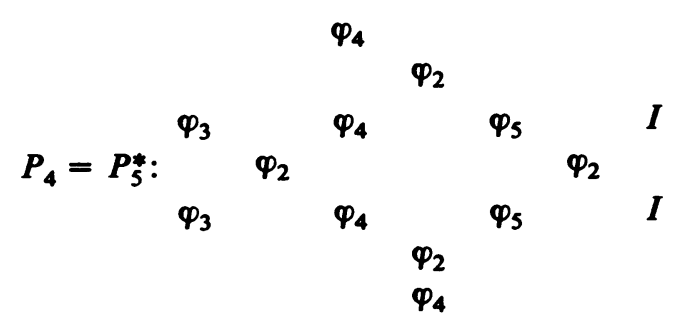

Finally combining Theorem 4.1 together with recent work of J. Alperin [1], K. Erdmann [5] and the authors [12] we show in $\$ 6$ that every indecomposable projective $B_{0}$-module $P$ of the principal 2-block $B_{0}$ of an arbitrary finite group $G$ with an abelian Sylow 2-subgroup $D$ of order $|D|=2^{n}$ has Loewy length $j(P)<$ $\max \left\{2 n+1,2^{n}\right\}$ (Theorem 6.1). This upper bound is sharp.

Concerning our terminology and notation we refer to Dornhoff [4], Feit [6], Gorenstein [8], and Green [9]. Discussions with L. Scott have been helpful.

1. Known results on the groups of Ree type. In this section we collect some known facts on the groups $R(q)$ of Ree type of order $|R(q)|=\left(q^{3}+1\right) q^{3}(q-1)$, where $q=3^{2 n+1}, m=3^{n}$, and $n=0,1,2, \ldots$ These results either can be found in Ward's paper [15] or are due to Fong [7].

Throughout this section, $(F, R, S)$ denotes a splitting 2-modular system for $R(q)$ and all its subgroups.

By $E_{n}$ we denote an elementary abelian group of order $n$. Let $E_{8}$ be a fixed Sylow 2-subgroup of $R(q)$ and fix an involution $1 \neq u \in E_{8}$. All involutions of $R(q)$ are conjugate.

If $U$ is a subgroup of $R(q)$, then $N(U)$ and $C(U)$ denote its normalizer and centralizer respectively in $R(q)$. By Ward [15], in each group $R(q)$ of Ree type the centralizer of the involution $u$ has the form

$$
C=C(u)=\langle u\rangle \times \operatorname{PSL}(2, q),
$$

where $q \equiv 3 \bmod 8$, and $E_{4}$ is a Sylow 2-subgroup of PSL $(2, q)$.

Let $N=N\left(E_{8}\right)$. Then $N$ is a holomorph of $E_{8}$ by the Frobenius group $F_{21}$ of order 21. Let $E$ be the normal subgroup of $N$ with index 3 . Then $E=E_{8} \cdot \mathbf{Z}_{7}$ is a Frobenius group, where $\mathbf{Z}_{r}$ denotes the cyclic group of order $r$. Furthermore,

$$
K=N \cap C=\langle u\rangle \times \mathfrak{A}_{4} .
$$


Let $P$ be a fixed Sylow 3-subgroup of $R(q)$ such that its normalizer $L=N(P)$ contains the involution $u$. By [15], $L$ is a holomorph of $P$ by $\langle u\rangle \times \mathbf{Z}_{(q-1) / 2}$, and

$$
H=L \cap C=\langle u\rangle \times\left(E_{q} \cdot \mathbf{Z}_{(q-1) / 2}\right),
$$

where $E_{q} \cdot \mathbf{Z}_{(q-1) / 2}$ is a Frobenius group. Furthermore, $C, L$ and $N$ are maximal subgroups, and

$$
T=L \cap N=\langle u\rangle \times \mathbf{Z}_{3} .
$$

Thus we have the following diagram of subgroups of $R(q)$ :

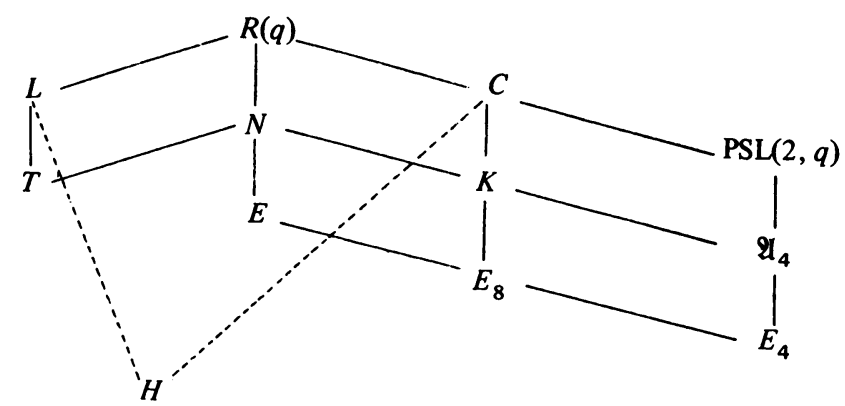

$I$ or $I_{G}$ denotes the trivial (modular) representation of any group $G$, and $P_{I}$ denotes the projective cover of $I$.

Throughout, $B=\left(B=e F G, \hat{B}=\hat{e} R G, B_{S}=\hat{B} \otimes_{R} S\right)$ denotes the principal 2-block of $R(q)$, where $e$ and $\hat{e}$ denote the block idempotents of $B$ and $\hat{B}$ respectively. As in [15], let $\xi_{i}, i=1,2, \ldots, 8, \xi_{1}=I$, denote the ordinary irreducible characters of $B$. We also let $\varphi_{i}, i=1,2, \ldots, 5, \varphi_{1}=I$, denote the Brauer characters of the corresponding simple $F R(q)$-modules.

Let $P_{i}$ be the projective cover of $\varphi_{i}, i=1,2, \ldots, 5, P_{1}=P_{I}$. In order to determine the socle series of $P_{i}$ we have to compute the three parameters $a, b$ and $c$ in Fong's [7] decomposition matrix of $B$ :

\begin{tabular}{c|c|c|c|c|c|c} 
character & $I$ & $\varphi_{2}$ & $\varphi_{3}$ & $\varphi_{4}$ & $\varphi_{5}$ & degree \\
\hline$I$ & 1 & 0 & 0 & 0 & 0 & 1 \\
\hline$\xi_{2}$ & 1 & 1 & 0 & 0 & 0 & $q^{2}-q+1$ \\
\hline$\xi_{3}$ & 1 & $a$ & 1 & $b$ & $b$ & $q^{3}$ \\
\hline$\xi_{4}$ & 1 & $a-1$ & 1 & $b$ & $b$ & $q\left(q^{2}-q-1\right)$ \\
\hline$\xi_{5}$ & 0 & $c$ & 0 & 1 & 0 & $\frac{1}{2}(q-1) m(q+1+3 m)$ \\
\hline$\xi_{7}$ & 0 & $c$ & 0 & 0 & 1 & $\frac{1}{2}(q-1) m(q+1+3 m)$ \\
\hline$\xi_{6}$ & 0 & $c-1$ & 0 & 1 & 0 & $\frac{1}{2}(q-1) m(q+1-3 m)$ \\
\hline$\xi_{8}$ & 0 & $c-1$ & 0 & 0 & 1 & $\frac{1}{2}(q-1) m(q+1-3 m)$ \\
\hline
\end{tabular}


Our computation of the integers $a, b$ and $c$ requires the following character tables and subsidiary results.

The principal 2-block of $C^{\prime} \cong \operatorname{PSL}(2, q)$ is denoted by $b_{0}=b_{0}\left(C^{\prime}\right)$. It has four ordinary characters $I, \chi_{q}, \chi^{\prime}$ and $\chi^{\prime \prime}$, and three modular characters $I, \chi^{\prime}$ and $\chi^{\prime \prime}$. Furthermore, $\chi^{\prime \prime}=\left(\chi^{\prime}\right)^{*}$. Let $P_{I}^{\prime}, P_{\chi^{\prime}}^{\prime}$ and $P_{\chi^{\prime \prime}}^{\prime}$ be the projective covers of the simple $b_{0}$-modules of $C^{\prime}$.

LEMMA 1.1. (a) The indecomposable projective modules of the principal block $b_{0}$ of $\operatorname{PSL}(2, q), q \equiv 3 \bmod 8$, have socle and Loewy series

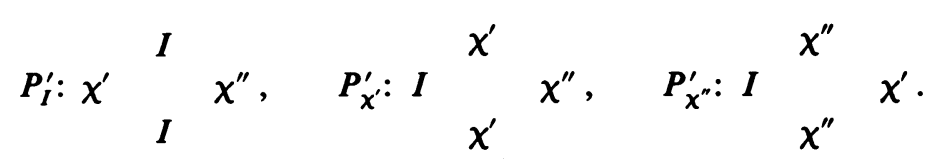

(b) The simple modules of $b_{0}$ may also be considered as the simple modules of $G=Z_{2} \times \operatorname{PSL}(2, q), q \equiv 3 \bmod 8$, in which group their projective covers have socle and Loewy series

$$
P_{I}: \begin{array}{llll}
\chi^{\prime} & I & \chi^{\prime \prime} \\
\chi^{\prime} & I & \chi^{\prime \prime} \\
& I
\end{array}, \quad P_{\chi^{\prime}}=\begin{array}{lll}
\chi^{\prime} & \begin{array}{l}
\chi^{\prime} \\
I \\
\chi^{\prime}
\end{array} & I \\
\chi^{\prime} & I
\end{array} \quad P_{\chi^{\prime \prime}}=\begin{array}{lll}
\chi^{\prime \prime} & \\
\chi^{\prime \prime} & \chi^{\prime} \\
\chi^{\prime \prime} & \chi^{\prime} \\
\chi^{\prime \prime} &
\end{array}
$$

(c) Consider again $\chi^{\prime}$ and $\chi^{\prime \prime}$ as $\operatorname{PSL}(2, q)$-modules. Then

$$
\chi_{\mid x_{4}}^{\prime}=1 \oplus \text { projectives, } \chi_{\mid x_{4}}^{\prime \prime}=1^{*} \oplus \text { projectives. }
$$

Proof. (a) See K. Erdmann [5]. ${ }^{3}$

(b) Since $G$ contains a central involution, this follows from (a) and Green's theorem (see Dornhoff [4, p. 329]).

(c) By K. Erdmann [5], 1 is the Green correspondent of $\chi^{\prime}$ in $\mathfrak{A}_{4}$, which is the normalizer of a Sylow 2-subgroup. Moreover, $\operatorname{PSL}(2, q)$ contains a dihedral subgroup of order $q-1$. Let $1,1^{-}$be the irreducible characters of the principal block of this group. Any other block is of defect 0 . We then compute

$$
\left(\chi^{\prime}, 1\right)=1, \quad\left(\chi^{\prime}, 1^{-}\right)=0,
$$

which shows that $\chi_{\mid \mathbf{Z}_{2}}^{\prime}=I \oplus$ projectives. Hence no component of $\chi_{\mid \mathbf{x}_{4}}^{\prime}$ has $\mathbf{Z}_{2}$ as a vertex. Since $\chi^{\prime \prime} \cong\left(\chi^{\prime}\right)^{*}$ assertion (c) follows.

In order to restate the character table of $C^{\prime}$ we use Ward's notation [15] for representatives of conjugacy classes of $C^{\prime}=\operatorname{PSL}(2, q)$. Thus the $R(q)$-conjugacy class of $u$ is identified with the one of $J$.

\footnotetext{
${ }^{3}$ This result is due to J. L. Alperin who announced it without details in his paper Minimal resolutions, Finite Groups 1972, North-Holland, Amsterdam, 1973, pp. 1-2. MR 50 \# 1045.
} 


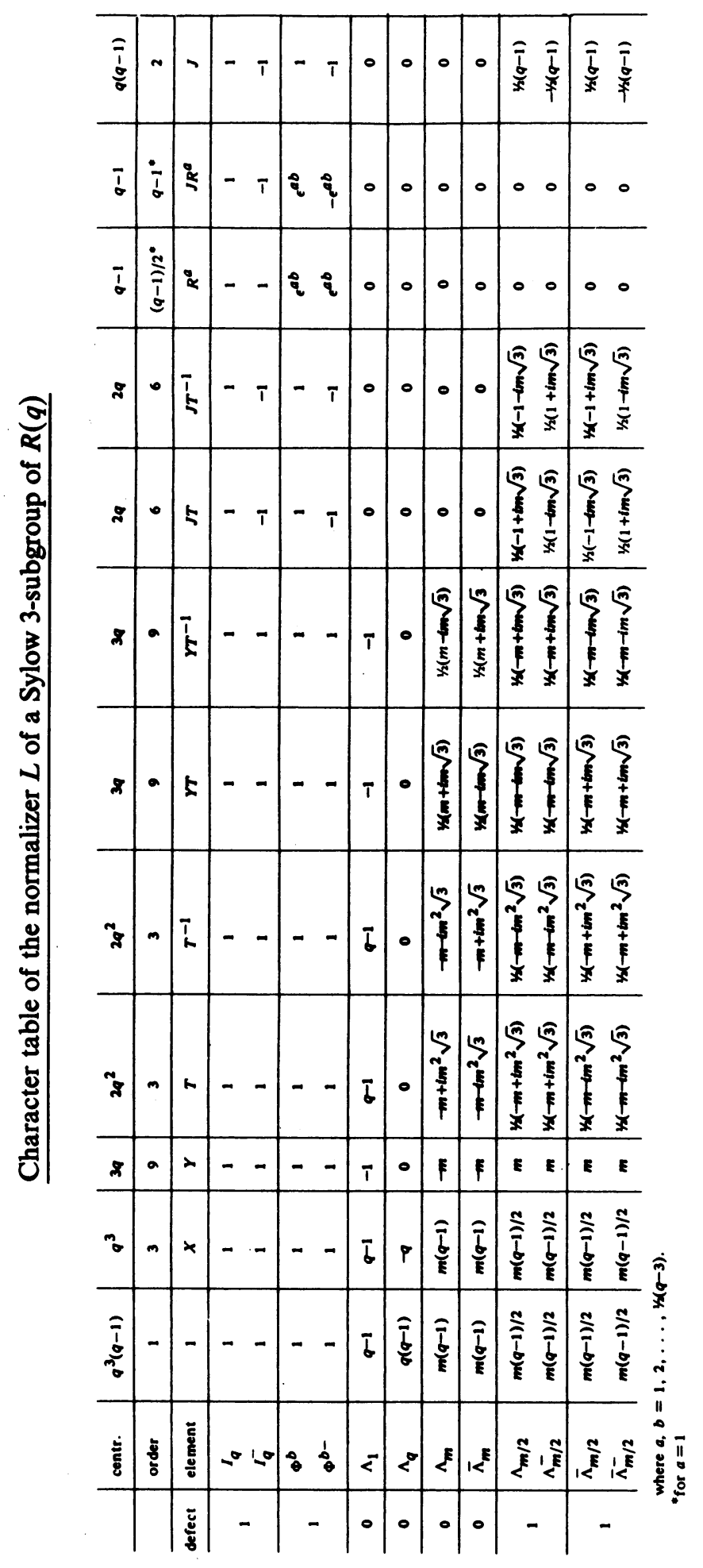


Character table of $C^{\prime}=\operatorname{PSL}(2, q)$

\begin{tabular}{c|c|c|c|c|c|c|c}
$\begin{array}{c}\text { central } \\
\text { index }\end{array}$ & 1 & $\frac{1}{2}\left(q^{2}-1\right)$ & $\frac{1}{2}\left(q^{2}-1\right)$ & $q(q+1)$ & $q(q-1)$ & $q(q-1)$ & $\frac{1}{2}(q(q-1))$ \\
\hline element & 1 & $T$ & $T^{-1}$ & $R^{h}$ & $s \delta_{0} j$ even & $S b_{0} j$ odd & $J$ \\
\hline$I$ & 1 & 1 & 1 & 1 & 1 & 1 & 1 \\
\hline$x_{q}$ & $q$ & 0 & 0 & 1 & -1 & -1 & -1 \\
\hline$\chi^{\prime}$ & $\frac{1}{2}(q-1)$ & $\frac{1}{2}(-1+\sqrt{-q})$ & $-\frac{1}{2}(1+\sqrt{-q})$ & 0 & -1 & 1 & 1 \\
\hline$x^{\prime \prime}$ & $\frac{1}{2}(q-1)$ & $-\frac{1}{2}(1+\sqrt{-q})$ & $\frac{1}{2}(-1+\sqrt{-q})$ & 0 & -1 & 1 & 1 \\
\hline$x_{k}$ & $q-1$ & -1 & -1 & 0 & $-\left(e^{j k}+e^{-j k}\right)$ & $-\left(e^{j k}+e^{-j k}\right)$ & $-2(-1)^{k}$ \\
\hline$\hat{x}_{1}$ & $q+1$ & 1 & 1 & $\omega^{h l}+\omega^{-h l}$ & 0 & 0 & 0 \\
\hline & & & & & & &
\end{tabular}

Where $1<h, j, k, l<r=\frac{1}{4}(q-3)$,

$$
\begin{gathered}
\varepsilon=e^{\pi i /(r+1)}, \quad \sum_{j=1}^{r} \varepsilon^{j k}+\varepsilon^{-j k}=-1-(-1)^{k}, \\
\omega=e^{2 \pi i /(2 r+1)}, \quad \sum_{l=1}^{r}\left(\omega^{h l}+\omega^{-h l}\right)=-1,
\end{gathered}
$$

all characters $\chi_{k}$ belong to blocks of defect 1 , and all characters $\hat{\chi}_{1}$ belong to blocks of defect zero of $C^{\prime}$.

From this character table and Ward's character table of $R(q)([15, \mathrm{pp} .87-88])$ we deduce

LEMMA 1.2. Let $\hat{e}$ be the block idempotent of the principal 2-block $\hat{B}$ of $R(q)$. Let $I$, $\chi^{\prime}, \chi^{\prime \prime}$ and $\chi_{q}$ be the ordinary irreducible characters of the principal block $b_{0}$ of $C^{\prime}=\operatorname{PSL}(2, q), q \equiv 3 \bmod 8$. Then:

(a) $I^{R(q)} \hat{e}=I \oplus \xi_{2} \oplus 3 \xi_{3} \oplus \xi_{4}$,

(b) $\chi^{\prime R(q)} \hat{e}=q \xi_{3} \oplus(q-2) \xi_{4} \oplus m \xi_{7} \oplus m \xi_{8}$,

(c) $\chi^{\prime \prime R(q)} \hat{e}=q \xi_{3} \oplus(q-2) \xi_{4} \oplus m \xi_{5} \oplus m \xi_{6}$,

(d) $\chi_{q}^{R(q)} e=2 \xi_{2} \oplus 2 q \xi_{3} \oplus 2 q \xi_{4} \oplus(m+1) \xi_{5} \oplus(m-1) \xi_{6} \oplus(m+1) \xi_{7} \oplus$ $(m-1) \xi_{8}$.

Using Fong's description [7, Lemma 2] of the Sylow 3-subgroup $P$ of $R(q)$, it is not difficult to compute the character table of its normalizer $L=N(P)$. In fact we show that it is uniquely determined although the structure of $P$ is not completely known. Again we use Ward's notation [15] of representatives of conjugacy classes. (See the Character table of the normalizer $L$ of a Sylow 3-subgroup of $R(q)$.)

From this and Ward's character table [15, pp. 87-88], we obtain

LEMMA 1.3. (a) $\xi_{2 \mid L}=I^{-}+\Lambda_{q}$.

(b) $\xi_{3 \mid L}=I+q \Lambda_{q}+m \Lambda_{m}+m \bar{\Lambda}_{m}+\Lambda_{1}+\frac{1}{2}(m+1) \Lambda_{m / 2}+\frac{1}{2}(m-1) \Lambda_{m / 2}^{-}$ $+\frac{1}{2}(m+1) \bar{\Lambda}_{m / 2}+\frac{1}{2}(m-1) \bar{\Lambda}_{m / 2}$.

(c) $\xi_{6 \mid L}=\frac{1}{2}(m-1) \Lambda_{q}+\Lambda_{m / 2}, \xi_{5 \mid L}=\frac{1}{2}(m+1) \Lambda_{q}+\Lambda_{m / 2}^{-}$.

(d) $\xi_{8 \mid L}=\frac{1}{2}(m-1) \Lambda_{q}+\bar{\Lambda}_{m / 2}, \xi_{7 \mid L}=\frac{1}{2}(m+1) \Lambda_{q}+\bar{\Lambda}_{m / 2}^{-}$. 
As in [12], centralizers of involutions play an important role in the course of the proofs. If $M$ is an $F G$-module or an $R G$-module and $u \neq 1$ is an involution of the finite group $G$, then

$$
\operatorname{ann}_{M}(1-u)=\{m \in M \mid m(1-u)=0\}
$$

is called the centralizer of $u$ in $M$.

If $X$ is an $R$-form of the irreducible character $\xi$ of the finite group $G$, and if $\bar{X}=X / X \pi$, where $\pi R$ is the maximal ideal of $R$, then we write

$$
z_{X}(\xi)=\operatorname{dim}_{F}\left(\operatorname{ann}_{\bar{X}}(1-u)\right) .
$$

For the irreducible characters $\xi_{2}$ and $\xi_{i}, i=5,6,7,8$, of the groups $R(q)$ of Ree type, this dimension $z_{X}(\xi)$ of a centralizer of the involution $u \neq 1$ is independent of the choice of the $R$-form $X$ by the following result.

LEMMA 1.4. (a) $z_{X}\left(\xi_{2}\right)=\frac{1}{2}\left(\xi_{2}(1)+1\right)$.

(b) $z_{X}\left(\xi_{i}\right)=\frac{1}{2}\left(\xi_{i}(1)+\frac{1}{2}(q-1)\right), i=5,6,7,8$.

(c) $\operatorname{dim}_{F}\left(\operatorname{ann}_{\Phi_{i}}(1-u)\right)=\frac{1}{4}(q-1)+\frac{1}{2} \operatorname{dim}_{F} \varphi_{i}, i=4,5$.

Proof. (a) Let $X$ be an $R$-form of $\xi_{2}$. Then Lemma 1.3(a) asserts that $\bar{X}_{\mid L}=\Lambda_{q}$ $\boxplus I$, because $\Lambda_{q}$ is a projective $F L$-module by the character table of $L$. Therefore,

$$
\begin{aligned}
z_{X}\left(\xi_{2}\right) & =\operatorname{dim}_{F}\left(\operatorname{ann}_{\bar{X}}(1-u)\right)=1+\frac{1}{2} \operatorname{dim} \Lambda_{q} \\
& =1+\frac{1}{2}\left(q^{2}-q\right)=\frac{1}{2}\left(\xi_{2}(1)+1\right) .
\end{aligned}
$$

(b) Let $X$ be an $R$-form of $\xi_{8}$. Then Lemma 1.3(d) asserts that $\bar{X}_{\mid L}=\frac{1}{2}(m-1) \Lambda_{q}$ $\oplus \bar{\Lambda}_{m / 2}$, because $\Lambda_{q}$ is a projective $F L$-module by the character table of $L$. Furthermore, $\bar{\Lambda}_{m / 2}$ belongs to a block of $L$ with defect group conjugate to $\langle u\rangle$. Now $H=C \cap L=C_{L}(u)=\langle u\rangle \times\left(E_{q} \cdot \mathbf{Z}_{(q-1) / 2}\right)$, and the second direct factor is a Frobenius group. Let $f$ be the Green correspondence between $L$ and $H$ with respect to $\langle u\rangle$. Then

$$
\bar{\Lambda}_{(m / 2) \mid H}=f\left(\bar{\Lambda}_{m / 2}\right) \oplus \text { projective } F H \text {-modules. }
$$

Thus $f\left(\bar{\Lambda}_{m / 2}\right)$ is an irreducible $F H$-module of degree $\frac{1}{2}(q-1)$ with $u$ in its kernel. Therefore (b) follows for $\xi_{8}$. By Lemma 1.3 the same argument applies to the other characters as well.

(c) Again let $X$ be an $R$-form of $\xi_{8}$. Then by Fong's decomposition matrix there is an integer $c$ such that $\bar{X}$ has composition factors $\varphi_{5}+(c-1) \varphi_{2}$. Since $\varphi_{2 \mid L}=\Lambda_{q}$ is a projective $F L$-module, it follows that

$$
\bar{X}_{\mid L}=\varphi_{5 \mid L} \oplus(c-1) \varphi_{2} .
$$

Hence from Lemma 1.3(d) and the Krull-Remak-Schmidt theorem we obtain

$$
\varphi_{s \mid L}=\frac{1}{2}(m+1-2 c) \Lambda_{q} \oplus \bar{\Lambda}_{m / 2}^{-} \text {. }
$$

As $\Lambda_{q}$ is projective, equation (*) now implies that

$$
\begin{aligned}
\operatorname{dim}_{F}\left(\operatorname{ann} \varphi_{5}(1-u)\right) & =\frac{1}{2}(q-1)+\frac{1}{2}\left(\operatorname{dim}_{F} \varphi_{5}-\frac{1}{2}(q-1)\right) \\
& =\frac{1}{4}(q-1)+\frac{1}{2} \operatorname{dim} \varphi_{5} .
\end{aligned}
$$


Hence (c) holds for $\varphi_{5}$. The same argument applies to $\varphi_{4}$, too.

For the sake of completeness we now restate the following inequality due to the first author [11]

LEMMA 1.5. Let $\bar{X}$ be a liftable FG-module of the form $\bar{X}=X / X \pi$. Let $\chi$ be the character of $X \otimes_{R} S$. If $u \neq 1$ is any involution of the finite group $G$, then

$$
\operatorname{dim}_{F}\left(\operatorname{ann}_{\bar{X}}(1-u)\right)>\frac{1}{2}[\chi(1)+|\chi(u)|] .
$$

Another subsidiary result is

LEMMA 1.6. Let $A$ be a finite-dimensional algebra over a field $F$. Let $S, T_{1}, T_{2}, \ldots, T_{n}$ be simple A-modules such that $\operatorname{dim}_{F} \operatorname{Ext}_{A}^{1}\left(T_{i}, S\right)=1$ for $i=$ $1,2, \ldots, n$. If the $A$-module $M$ has a submodule $M_{0} \simeq r S$ such that $M / M_{0} \simeq T_{1} \oplus$ $T_{2} \oplus \cdots \oplus T_{n}$, where $r \geqslant 2$, then $M$ is not indecomposable, and every direct summand has simple socle.

Proof. By induction we may assume that $r=2$. Hence $M_{0}=S_{1} \oplus S_{2}=\operatorname{soc} M$, where $S_{i} \cong S$ for $i=1,2$. Let $M_{1}=M / S_{1}$. Then $M_{1}$ is a direct sum of a semisimple $A$-module $U_{1}$ and an $A$-module $V_{2}$ with soc $V_{2}=S_{2}$ and $V_{2} / S_{2} \simeq T_{i_{1}}$ $\oplus \cdots \oplus T_{i_{k}}$. Let $U$ be the preimage of $U_{1}$ and $V$ the one of $V_{2}$. Then our hypothesis implies that $U$ has simple socle soc $U=S_{1}$ and that $V \simeq V_{2} \oplus S_{1}$. Hence $V$ has a direct sum decomposition $V=V_{1} \oplus S_{1}$, where $V_{2} \simeq V_{1}+S_{1} / S_{1}$ $\cong V_{1}$. Thus $M=U \oplus V_{1}$.

We complete this section by restating some useful definitions and notations of [12].

Let $G$ be an arbitrary finite group. For any pair of $F G$-modules $X, Y$ and a subgroup $U$ of $G$, denote $(X, Y)_{U}:=\operatorname{Hom}_{F U}(X, Y) . X \circ Y$ denotes any extension of $X$ by $Y$, so that there exists an exact sequence $0 \rightarrow Y \rightarrow X \circ Y \rightarrow X \rightarrow 0 . \Omega X$ denotes the Heller module of $X$, so that there exists an exact sequence $0 \rightarrow \Omega X \rightarrow P$ $\rightarrow X \rightarrow 0$, whenever $P$ is a projective cover of $X$.

$(X, Y)_{1, G}$ consists of all $F G$-module homomorphisms from $X$ into $Y$ factorizing through a projective $F G$-module $P$, and

$$
(X, Y)_{G}^{1}=(X, Y)_{G} /(X, Y)_{1, G}
$$

Definition. Let $G$ be a finite group, $p|| G \mid$ a prime number, and $F$ a splitting field for all subgroups of $G$ of characteristic $p>0$. Let $H$ be a subgroup of $G$, and $U$ an indecomposable $F G$-module which is $H$-projective. If a component $f^{*}(U)$ of $U_{H}$ is the only direct summand $E$ of $U_{H}$ satisfying $U \mid E^{G}$, then $f^{*}(U)$ is called a generalized Green correspondent of $U$ in $H$.

2. The Green correspondents of $\varphi_{2}, \varphi_{4}$ and $\varphi_{5}$. In this section we determine the Green correspondents of the simple $F R(q)$-modules $\varphi_{i}, i \in\{2,4,5\}$, of the principal 2-block $B$ of the groups $R(q)$ of Ree type. We also show that $c=1$.

In order to derive these assertions we study the structure of two permutation modules. Let $U=\left(I_{L}\right)^{R(q)}$ be the permutation module belonging to the 2 -fold transitive permutation representation of $R(q)$, see Ward [15]. Then $\hat{U} \otimes_{R} S=I \oplus$ $\xi_{3}$, and $U$ is a self-dual indecomposable $F R(q)$-module belonging to $B$ with the 
following properties, of which assertion (d) is due to L. Scott [14].

LEMMA 2.1. (a) $S(U)=I \cong U / U J$.

(b) $\operatorname{Ext}_{F R(q)}^{1}(I, I)=0=\operatorname{Ext}_{F R(q)}^{2}(I, I)$.

(c) $S_{2}(U) / S(U)=\varphi_{2}$.

(d) The projective cover $P_{1}$ of $I$ has a submodule $\varphi_{2}$.

(e) $U$ has vertex $\operatorname{vx}(U)={ }_{R(q)}\langle u\rangle=E_{2}$.

(f) $U$ has a minimal projective resolution $0 \leftarrow U \leftarrow P_{I} \leftarrow \Omega U \leftarrow 0$, and $U \simeq \Omega U$.

Proof. As $U$ is a permutation module, it is liftable, self-dual, $I$ occurs in the head $U / U J$ and in the socle of $U$. Since $\hat{U} \otimes_{R} S=I \oplus \xi_{3}, \operatorname{dim}_{F} \operatorname{End}_{F G}(U)=2$. If $U$ were not indecomposable, then $\operatorname{End}_{F G}(U)=F \oplus F$, and $I$ would be a direct summand of $U$. However, $E_{2}=\langle u\rangle$ is a Sylow 2-subgroup of $L$, which implies that each component of $U$ is $E_{2}$-projective. But $\operatorname{vx}(I)={ }_{R(q)} E_{8}$. Hence $U$ is indecomposable, and its vertex $\operatorname{vx}(U)={ }_{R(q)} E_{2}$. Furthermore, $I=\operatorname{soc}(U) \simeq U / U J$. Thus (a) and (e) hold.

By [9], the Heller operator $\Omega$ commutes with induction from $L$ to $R(q)$. Hence (f) follows.

As $N$ is also the normalizer of the Sylow 2-subgroup of the smallest Janko group $J_{1}$, assertion (b) follows from [12], Lemma 1.1 and the proof of Lemma 6.3.

By Fong's decomposition matrix of $B$ the character $\xi_{2}$ has an $R$-form $X$ such that $\bar{X}=X / X \pi={ }_{I}$. As $\operatorname{Ext}_{F R(q)}^{2}(I, I)=0$ by (b), the argument of L. Scott [14] yields the existence of the uniserial module

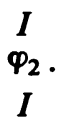

Thus (d) holds.

Furthermore, the same reasoning shows the existence of

$$
T_{i}=\stackrel{I}{\varphi_{i}},
$$

whenever ${ }_{I}^{\varphi_{1}}$ belongs to the second socle $S_{2}(U)$ of $U$. Since $\varphi_{3}$ has multiplicity 1 in $U$ by (f) and Fong's decomposition matrix

$$
U \approx \begin{gathered}
I \\
\varphi_{2} . \\
I
\end{gathered}
$$

Therefore by (a) and (d), its Loewy length $j(U)>3$. If $T_{i}$ were a submodule of $U$, then it would therefore be a proper submodule of $U$. Hence the multiplicity of $I$ in $U$ would be at least 3 by (a). Thus by (f), the Cartan invariant $c_{11}>2 \cdot 3=6$. But $c_{11}=4$ by Fong's decomposition matrix. This contradiction proves (c) and completes the proof.

Besides the permutation module $U=\left(I_{L}\right)^{R(q)}$ we consider the component $W$ in the principal block $B \leftrightarrow e$ of the permutation module $\left(I_{C}\right)^{R(q)}$, i.e. $W=\left(I_{C}\right)^{R(q)} e$. 
Before we can determine its main properties, we have to study the restrictions of $\varphi_{2}$ to $C$ and $L$.

LEMMA 2.2. (a) $\varphi_{2 \mid L}=\Lambda_{q}$ is a simple and projective $F L$-module.

(b) If $e_{0}$ denotes the block idempotent of the principal 2-block $b_{0}$ of $C$, then $\varphi_{2 \mid c} e_{0}$ is indecomposable and has socle series

$$
\varphi_{2 \mid c} e_{0}=\begin{array}{lll}
I & \chi^{\prime} & \chi^{\prime \prime} \\
I & \chi^{\prime} & \chi^{\prime \prime}
\end{array}
$$

where $I, \chi^{\prime}$, and $\chi^{\prime \prime}$ are the simple $F C$-modules of $b_{0}$. Furthermore, its vertex is $E_{8}$.

(c) $\varphi_{2 \mid c}\left(1-e_{0}\right)$ is a projective FC-module.

(d) If $e_{0}$ also denotes the principal 2-block idempotent of $C^{\prime}$, then also $\varphi_{2 \mid C^{\prime}} e_{0}$ is indecomposable with $E_{4}$ as a vertex and has socle series

$$
\varphi_{2 \mid C^{\prime}} e_{0}=\begin{array}{lll}
I & \chi^{\prime} & \chi^{\prime \prime} \\
I & \chi^{\prime} & \chi^{\prime \prime}
\end{array}
$$

(e) $\varphi_{2 \mid C^{\prime}}\left(1-e_{0}\right)$ is a projective $F C^{\prime}$-module.

Proof. (a) holds by Lemma 1.3(a), Fong's decomposition matrix of $B$, and the character table of $L$.

(b) Since every nonprincipal 2-block of $C^{\prime}$ has defect at most 1 , any nonprojective component $V$ of $\varphi_{2 \mid C^{\prime}}\left(1-e_{0}\right)$ has vertex $\operatorname{vx}(V)={ }_{C^{\prime}} E_{2}$. However all involutions of $R(q)$ are conjugate. Hence $V$ is projective by (a). Thus $V=0$.

(c) is an immediate consequence of (e) by Nagao's lemma, see Dornhoff $[4, p$. 353].

(d) By Lemma 1.2, $A^{\prime}=\varphi_{2 \mid C^{\prime}} e_{0}$ has composition factors $2 I+2 \chi^{\prime}+2 \chi^{\prime \prime}$. Since all involutions of $R(q)$ are conjugate, it follows from (a) that $\left.A^{\prime}\right|_{\langle v\rangle}$ is projective for every involution $v$ of $C^{\prime}$. Therefore the sources of the components of $A^{\prime}$ are even-dimensional. Hence every component of $A^{\prime}$ has an even number of composition factors. As $\varphi_{2}$ is self-dual and algebraically invariant, Lemma 1.1 implies that $A^{\prime}$ is indecomposable and projective free. Therefore $\operatorname{vx}\left(A^{\prime}\right)=E_{4}$, and (d) follows.

(e) Certainly $A=\varphi_{2 \mid C} e_{0}$ is indecomposable by (d). By Knörr's theorem [10], $\operatorname{vx}\left(\varphi_{2}\right)={ }_{R(q)} E_{8}$. Hence $E_{8}$ is a vertex of $A$. Furthermore, (a) implies

$$
\operatorname{dim}_{F} \operatorname{ann}_{A}(1-u)=\frac{1}{2} \operatorname{dim} A .
$$

Again the self-duality and algebraic invariance of $A$ imply that $\operatorname{ann}_{A}(1-u)$ is semisimple. Hence (b) follows, because $\operatorname{soc} A=\operatorname{ann}_{A}(1-u)$. This completes the proof of Lemma 2.2 .

LEMMA 2.3. The $F R(q)$-module $W=\left(I_{C}\right)^{R(q)}$ e of the principal 2-block $B \leftrightarrow e$ of $R(q)$ has the following properties:

(a) $\hat{W} \otimes_{R} S=I \oplus 2 \xi_{3}$,

(b) $\operatorname{End}_{F R(q)}(W) \simeq \operatorname{End}_{R R(q)}(\hat{W}) / \pi \operatorname{End}_{R R(q)}(\hat{W})$,

(c) $\operatorname{dim}_{F} \operatorname{End}_{F R(q)}(W)=5$,

(d) $\operatorname{dim}_{F} \operatorname{Hom}_{F R(q)}(U, W)=3=\operatorname{dim}_{F} \operatorname{Hom}_{F R(q)}(W, U)$,

(e) $W=I \oplus V \oplus V^{*}$, where the dual $B$-modules $V$ and $V^{*}$ have socles soc $V=$ $\varphi_{2}$ and $\operatorname{soc} V^{*}=\varphi_{3}$. 
Proof. (a) follows immediately from Ward's character table [15] and the Frobenius relations.

(b) $Y=\left(I_{C}\right)^{R(q)}$ is a permutation module. Thus,

$$
\operatorname{End}_{F R(q)}(Y) \cong \operatorname{End}_{R R(q)}(Y) / \pi \operatorname{End}_{R R(q)}(Y) \text {. }
$$

Since $\hat{W}=\hat{Y} \hat{e}$,

$$
\operatorname{End}_{R R(q)}(\hat{W}) \simeq \hat{e}\left[\operatorname{End}_{R R(q)}(\hat{Y})\right] \hat{e}
$$

which implies (b).

(c) follows from (a) and (b).

(d) As $\hat{U} \otimes_{R} S=I \oplus \xi_{3}$ we obtain from (a) that

$\operatorname{dim}_{S} \operatorname{Hom}_{S R(q)}\left(\hat{U} \otimes_{R} S, \hat{W} \otimes_{R} S\right)=3=\operatorname{dim}_{S} \operatorname{Hom}_{S R(q)}\left(\hat{W} \otimes_{R} S, \hat{U} \otimes_{R} S\right)$

Since $U$ and $Y$ are permutation modules, it follows that

$$
\operatorname{Hom}_{F R(q)}(U, W) \cong \operatorname{Hom}_{R R(q)}(\hat{U}, \hat{W}) / \pi \operatorname{Hom}_{R R(q)}(\hat{U}, \hat{W}) \text {, }
$$

because $\hat{W}=\hat{Y} \hat{e}$. Interchanging $U$ and $W$ we obtain the other isomorphism. Thus,

$$
\operatorname{dim}_{F} \operatorname{Hom}_{F R(q)}(U, W)=3=\operatorname{dim}_{F} \operatorname{Hom}_{F R(q)}(W, U) \text {. }
$$

(e) As $K=N \cap C$ we have a Green correspondence $g_{1}$ between $K$ and $C$ with respect to $E_{8}$. Since $g_{1}(I)=I$,

$$
\left(I_{K}\right)^{C} \approx I_{C} \oplus Q,
$$

where $Q$ is a direct sum of indecomposable $F C$-modules with vertices contained in Klein four subgroups of $C$.

The index $|N: K|=7$. Thus $\left(I_{K}\right)^{N}$ has composition factors $I, 3$ and $3^{*}$ by [12]. Clearly $E_{8}$ is normal in $N$ and has odd index. Therefore $\left(I_{E_{8}}\right)^{N}$ is a semisimple $F N$-module. As $I_{K}$ has vertex $E_{8}$ it is a component of $\left(I_{E_{8}}\right)^{K}$. Hence $\left(I_{K}\right)^{N}$ is a direct summand of the semisimple $F N$-module $\left(I_{E_{8}}\right)^{N}$, which implies

$$
\left(I_{K}\right)^{N} \cong I_{N} \oplus 3 \oplus 3^{*} \text {. }
$$

Now let $g$ be the Green correspondence between $N$ and $R(q)$ with respect to $E_{8}$. Then

$$
\left(I_{K}\right)^{R(q)} \simeq I \oplus g(3) \oplus g\left(3^{*}\right) \oplus A,
$$

where $A$ is a direct sum of indecomposable $F R(q)$-modules with smaller vertices than $E_{8}$. So

$$
\left(I_{K}\right)^{R(q)}=\left(\left(I_{K}\right)^{C}\right)^{R(q)} \simeq\left(I_{C}\right)^{R(q)} \oplus Q^{R(q)}
$$

yields

$$
W=\left(I_{C}^{R(q)}\right) e \cong I \oplus g(3) \oplus g\left(3^{*}\right) \oplus W_{0}
$$

As

$$
\operatorname{dim}_{F}\left(\operatorname{End}_{F R(q)}\left(I \oplus g(3) \oplus g\left(3^{*}\right)\right)>5,\right.
$$

(c) implies $W_{0}=0$. Hence $W=I \oplus V \oplus V^{*}$ for some indecomposable $F R(q)$ module $V$. 
By Lemma 2.2(b) $\varphi_{2}$ occurs once in the socle of $W$. Hence $V$ and $V^{*}$ are algebraically invariant. As $\operatorname{dim}_{F} \operatorname{End}_{F R(q)}(W)=5$ it follows that neither $\varphi_{4}$ nor $\varphi_{5}$ occurs in the socle of $W$. Furthermore, we may assume without loss of generality that $\operatorname{soc} V=\varphi_{2}$. Hence soc $V^{*}=\varphi_{3}$ which completes the proof.

Proposition 2.4. Let $e_{0}$ be the block idempotent of the principal 2-block $b_{0}$ of $C$. Then:

(a) $c=1$,

(b) $\varphi_{4 \mid C} e_{0}=\chi^{\prime \prime} \oplus \frac{1}{2}(m-1) P_{\chi^{\prime \prime}}$,

(c) $\varphi_{5 \mid C} e_{0}=\chi^{\prime} \oplus \frac{1}{2}(m-1) P_{\chi^{\prime}}$.

Proof. Let $T$ be an $R$-form of $\xi_{8}$. Then by Fong's decomposition matrix, $\bar{T}=T / T \pi$ has composition factors $\varphi_{5}+(c-1) \varphi_{2}$. By Lemma $2.2(\mathrm{~b}), \varphi_{2 \mid c} e_{0}$ has composition factors $2 I+2 \chi^{\prime}+2 \chi^{\prime \prime}$. Therefore Lemma 1.2 implies that $\varphi_{5 \mid C} e_{0}$ has composition factors

$$
(m+1-2 c) I+(2 m+1-2 c) \chi^{\prime}+(m+1-2 c) \chi^{\prime \prime} .
$$

Since the involution $u \neq 1$ is in the center of $C=\langle u\rangle \times C^{\prime}, Y=\operatorname{ann}_{\varphi_{s \mid c}}(1-u)$ is an $F C$-submodule of $\varphi_{5 \mid C}$. As $u$ acts trivially on $Y$, it follows that $Y$ is an $F C^{\prime}$-module. In particular, every nonprojective component of $Y$ has Loewy length 2 , because $C^{\prime} \cong \operatorname{PSL}(2, q), q \equiv 3 \bmod 8$. Since $I_{C}$ does not occur in the socles of $\varphi_{5 \mid C}$ and $\varphi_{4 \mid C}$ by Lemma 2.3 , it follows that $I_{C}$ does not occur in the socle and the head of $Y$, because $\varphi_{4}^{*}=\varphi_{5}$. Hence by Donovan-Freislich [3] every indecomposable summand $U$ of $Y e_{0}$ is isomorphic to one of the following indecomposable $F C^{\prime}$-modules:

$$
\chi^{\prime}, \chi^{\prime \prime}, \frac{\chi^{\prime}}{\chi^{\prime \prime}}, \frac{\chi^{\prime \prime}}{\chi^{\prime}}, P_{\chi^{\prime}}^{\prime} \text { and } P_{\chi^{\prime \prime}}^{\prime}
$$

Let $r, s, t$ and $u$ be the multiplicities of $\chi^{\prime}, \chi^{\prime \prime}, \chi^{\prime \prime}$ and $\chi^{\prime \prime} \chi^{\prime \prime}$ respectively in $Y e_{0}$, and let $p$ be the number of indecomposable projective summands of $Y e_{0}$. Then

$$
\operatorname{dim}_{F} Y e_{0}=p\left(q+\frac{1}{2}(q-1)\right)+\frac{1}{2}(r+s)(q-1)+(u+t)(q-1) .
$$

Hence by Lemma 1.1, the multiplicity of the composition factor $I_{C}$ in $Y e_{0}$ is $p$.

Let $X=(1-u) \varphi_{5 \mid C}$. Then $X \subseteq Y$, and by Lemma 1.4(c),

$$
\operatorname{dim}_{F}(Y / X)=\frac{1}{2}(p-1) \text {. }
$$

As the multiplicity of $I_{C}$ in $\varphi_{5 \mid C}$ is

$$
m+1-2 c=\frac{1}{3} \sqrt{3 q}+1-2 c<\frac{1}{2}(q-1),
$$

it follows from the character table of $\operatorname{PSL}(2, q)$ that $Y / X$ is isomorphic to $\chi^{\prime}$ or $\chi^{\prime \prime}$. Thus,

$$
Y / X \simeq Y e_{0} / X e_{0} .
$$

As $\varphi_{5} e_{0} / Y e_{0} \cong X e_{0}$, it follows that $I_{C}$ occurs $\frac{1}{2}(m+1-2 c)$ times as a composition factor of $Y e_{0}$. Hence $p=\frac{1}{2}(m+1-2 c)$. 
By (*),

$$
\operatorname{dim}_{F} \varphi_{5} e_{0}=(m+1) q+\frac{m}{2}(q-1)-2 c q .
$$

Hence (***) implies

$$
\operatorname{dim}_{F} Y e_{0}=\frac{m+1}{2} q+\frac{m+1}{4}(q-1)-c q .
$$

Inserting the value of $p$ into (***) we also obtain $\operatorname{dim}_{F} Y e_{0}=\frac{1}{2}(m+1-2 c)\left(q+\frac{1}{2}(q-1)\right)+\frac{1}{2}(r+s)(q-1)+(u+t)(q-1)$.

Thus,

$$
\frac{1}{2}(q-1) c=\frac{1}{2}(r+s)(q-1)+(u+t)(q-1),
$$

and

$$
c=r+s+2(u+t) \text {. }
$$

Let $x$ be the multiplicity of the direct summand $P_{x^{\prime}}^{\prime}$ in $Y e_{0}$ and let $y$ be the one of $P_{x^{\prime \prime}}^{\prime}$ Then

$$
x+y=p=\frac{1}{2}(m+1-2 c) .
$$

By Lemma 1.1, the multiplicity of $\chi^{\prime \prime}$ in $x P_{x^{\prime}}^{\prime} \oplus y P_{x^{\prime \prime}}^{\prime}$ is $x+2 y$. Since (*) and the simplicity of $Y e_{0} / X e_{0}$ imply that $\chi^{\prime \prime}$ occurs $\frac{1}{2}(m+1-2 c)$ times as a composition factor of $Y e_{0}$, it follows that

$$
x+2 y<\frac{1}{2}(m+1-2 c) .
$$

Therefore $y=0$, and $x=\frac{1}{2}(m+1-2 c)$. Hence Lemma 1.1 asserts that $t=u=s$ $=0$, and $c=r$. Thus

$$
Y e_{0}=c \chi^{\prime} \oplus \frac{1}{2}(m+1-2 c) P_{\chi^{\prime \prime}}^{\prime}
$$

Since $I_{C}$ is not contained in the head of $X e_{0}$, it follows from (***) that

$$
\varphi_{5} e_{0} / Y e_{0} \cong X e_{0} \cong(c-1) \chi^{\prime} \oplus \frac{1}{2}(m+1-2 c) P_{\chi^{\prime}}^{\prime}
$$

Restricting $\varphi_{5}$ now to $C^{\prime}$ we obtain

$$
A_{1}=\varphi_{5 \mid C^{\prime}} e_{0}=(2 c-1) \chi^{\prime} \oplus(m+1-2 c) P_{\chi^{\prime}}^{\prime}
$$

Let $v \neq 1$ be an involution of $C^{\prime}$, and let $z_{A_{1}}(v)=\operatorname{dim}_{F}\left(\operatorname{ann}_{A_{1}}(1-v)\right)$. Since by Lemma 1.1, the Green correspondent $f\left(\chi^{\prime}\right)$ of $\chi^{\prime}$ in $\mathfrak{X}_{4}$ is one-dimensional and has a projective complement, we obtain

$$
\begin{aligned}
z_{A_{1}}(v) & =2 c-1+\frac{1}{2}(2 c-1)\left(\operatorname{dim}_{F} \chi^{\prime}-1\right)+\frac{1}{2}(m+1-2 c) \operatorname{dim}_{F} P_{X^{\prime}} \\
& =\frac{1}{2}\left(2 c-1+\operatorname{dim} A_{1}\right) .
\end{aligned}
$$

We now choose the $R$-form $T$ of $\xi_{8}$ so that $\varphi_{5}=\operatorname{soc}(\bar{T})$, see [4, Lemma 68.10], due to J. Thompson. By Lemma 1.3, $\bar{T}$ has the following composition factors:

$$
\begin{aligned}
\left.\bar{T}\right|_{C^{\prime}}= & (m-1) I+(2 m-1) \chi^{\prime}+(m-1) \chi^{\prime \prime} \\
& +m \sum_{s=1}^{r / 2} \chi_{2 s-1}+(m-2) \sum_{s=1}^{r / 2} \chi_{2 s}+(m-1) \sum_{l=1}^{r} \hat{\chi}_{l} .
\end{aligned}
$$


Lemma 1.4(b) asserts

$$
z_{T}\left(\xi_{8}\right)=\frac{1}{2}\left(\xi_{8}(1)+\frac{1}{2}(q-1)\right)=\frac{1}{4}[m(q-1)(q+1-3 m)+q-1] .
$$

Let $T_{1}=\left.\bar{T}\right|_{C^{\prime}} e_{0}$ and $T_{2}=\left.\bar{T}\right|_{C^{\prime}}\left(1-e_{0}\right)$, where $e_{0}$ denotes the block idempotent of the principal 2-block of $C^{\prime}$. By Lemma 1.5,

$$
z_{T_{1}}(v)>\frac{1}{2}\left(\frac{1}{2} m(q-1)+(m-1) q+1\right)=c_{1},
$$

because $T_{1}$ has odd dimension. Likewise,

$$
\begin{aligned}
z_{T_{2}}(v) & >\frac{1}{8}[(m-2)(q-1)(q-3)+(m-1)(q+1)(q-3)+(q+1)(q-3)] \\
& =c_{2} .
\end{aligned}
$$

An easy computation shows

$$
z_{T}\left(\xi_{8}\right)=z_{T_{1}}(v)+z_{T_{2}}(v)>c_{1}+c_{2}=z_{T}\left(\xi_{8}\right) .
$$

Hence $z_{T_{1}}(v)=c_{1}=\frac{1}{2}\left(\operatorname{dim}_{F} T_{1}+1\right)$. Since $\bar{T}$ has composition factors $\varphi_{5}+$ $(c-1) \varphi_{2}$, and as $\varphi_{2 \mid\langle v\rangle}$ is projective,

$$
\begin{aligned}
z_{T_{1}}(v) & =z_{A_{1}}(v)+(c-1) \operatorname{dim}_{F} \operatorname{ann}_{\varphi_{2 \mid c}} e_{0}(1-v) \\
& =z_{A_{1}}(v)+(c-1) q \\
& =\frac{1}{2}\left(2 c-1+\operatorname{dim} A_{1}\right)+(c-1) q \\
& =\frac{1}{2}\left(2 c-1+\operatorname{dim}_{F} T_{1}\right) .
\end{aligned}
$$

Thus $2 c-1=1$, and $c=1$, which proves (a).

Furthermore, $\varphi_{5 \mid C^{\prime}} e_{0}=A_{1}=\chi^{\prime} \oplus(m-1) P_{\chi^{\prime}}^{\prime}$, and $Y e_{0}=\chi^{\prime} \oplus \frac{1}{2}(m-1) P_{\chi^{\prime}}^{\prime}$. As the involution $u$ acts trivially on $\chi^{\prime}$ and as $C=\langle u\rangle \times C^{\prime}$, it follows from the decomposition of $A_{1}$ that $\chi^{\prime}$ is a direct summand of $\varphi_{5 \mid C} e_{0}=\chi^{\prime} \oplus U$, where $U$ denotes a complement of $\chi^{\prime}$ in $\varphi_{s \mid C} e_{0}$. Since $\varphi_{s \mid C} e_{0}$ and $Y e_{0}$ have the same socle, they also have the same injective hull, which is a direct sum of $\frac{1}{2}(m+1)$ copies of $P_{\chi^{\prime}}$. By Lemma 1.1, $U$ and $\frac{1}{2}(m-1) P_{\chi^{\prime}}$ have the same composition factors including multiplicities. Thus $U=\frac{1}{2}(m-1) P_{\chi^{\prime}}$, which proves (c). Now (b) follows by duality. This completes the proof of Proposition 2.4 .

Proposition 2.5. The simple FR(q)-modules $\varphi_{2}, \varphi_{4}$ and $\varphi_{5}$ have Green correspondents with the following socle and Loewy series:

(a) $f\left(\varphi_{2}\right)=3_{3}^{*}$, and $\varphi_{2 \mid N}=f\left(\varphi_{2}\right) \oplus$ projectives.

(b) $f\left(\varphi_{4}\right)=1^{*}$, and $\varphi_{4 \mid N}=f\left(\varphi_{4}\right) \oplus E_{2}$-projectives.

(c) $f\left(\varphi_{5}\right)=1$, and $\varphi_{5 \mid N}=f\left(\varphi_{5}\right) \oplus E_{2}$-projectives.

Proof. (a) By Lemma 2.2(b) and (c),

$$
\varphi_{2 \mid C}=\begin{array}{lll}
I & \chi^{\prime} & \chi^{\prime \prime} \\
I & \chi^{\prime} & \chi^{\prime \prime}
\end{array} \oplus \text { projectives, }
$$

where the nonprojective summand is indecomposable with vertex $E_{8}$. By Lemmas 1.1(c) and 2.2(d),

$$
\varphi_{2 \mid x_{4}}=\begin{array}{lll}
I & 1 & 1^{*} \\
I & 1 & 1^{*}
\end{array} \text { projectives, }
$$


where the nonprojective summand is indecomposable with vertex $E_{4}$. Using the argument of Lemma 2.2(b), it follows that

$$
\varphi_{2 \mid K}=\begin{array}{lll}
I & 1 & 1^{*} \\
I & 1 & 1^{*}
\end{array} \text { projectives, }
$$

where the nonprojective summand is indecomposable with vertex $E_{8}$. As $K=N \cap$ $C$ and $N$ is 2-closed,

$$
f\left(\varphi_{2}\right)_{\mid K}=\begin{array}{lll}
I & 1 & 1^{*} \\
I & 1 & 1^{*}
\end{array}
$$

Hence self-duality of $f\left(\varphi_{2}\right)$ implies that its composition factors are $2 I+2 \cdot 1^{*}+2$ - 1 or $3+3^{*}$. If the first possibility occurs, an element of order 7 is in the kernel of $f\left(\varphi_{2}\right)$. Thus the kernel of $f\left(\varphi_{2}\right)$ contains $E$. However, $E_{8}$ is not in kernel, since $f\left(\varphi_{2}\right)_{\mid E_{2}}$ is projective. Therefore $f\left(\varphi_{2}\right)$ equals $3^{3}$ or $3_{3}^{*}$, as its Loewy length is 2 . Now

$$
\varphi_{2 \mid N}=f\left(\varphi_{2}\right) \oplus \text { projective }
$$

by (*). Hence Lemma 1.1 of [12] and Lemma $2.1(d)$ imply that

$$
Y=f\left(\begin{array}{c}
I \\
\varphi_{2} \\
I
\end{array}\right)
$$

is an indecomposable $F N$-module. Since $Y$ is the only nonprojective summand of $I$
$\varphi_{2}$ , it is not projective. Thus Proposition 2.3 of [12] implies $f\left(\varphi_{2}\right)=\frac{3^{*}}{3}$.

(b) By Proposition 2.4(b) and Lemma 1.1(c), $\varphi_{4 \mid K}$ has only one summand with vertex $E_{8}$, namely 1 . As in the proof of (a), we obtain $f\left(\varphi_{4}\right)_{\mid K}=1$. Thus $f\left(\varphi_{4}\right)=1$.

(c) follows now from (b) by duality.

3. The decomposition matrix of $B$. In this section we determine the parameters $a$ and $b$ of Fong's decomposition matrix of the principal 2-block $B$ of the groups $R(q)$ of Ree type.

\section{LEMMA 3.1. $B$ has Cartan matrix}

\begin{tabular}{c|c|c|c|c|c} 
& $I$ & $\varphi_{2}$ & $\varphi_{3}$ & $\varphi_{4}$ & $\varphi_{5}$ \\
\hline$I$ & 4 & $2 a$ & 2 & $2 b$ & $2 b$ \\
\hline$\varphi_{2}$ & $2 a$ & $2 a^{2}-2 a+4$ & $2 a-1$ & $2 a b-b+1$ & $2 a b-b+1$ \\
\hline$\varphi_{3}$ & 2 & $2 a-1$ & 2 & $2 b$ & $2 b$ \\
\hline$\varphi_{4}$ & $2 b$ & $2 a b-b+1$ & $2 b$ & $2 b^{2}+2$ & $2 b^{2}$ \\
\hline$\varphi_{5}$ & $2 b$ & $2 a b-b+1$ & $2 b$ & $2 b^{2}$ & $2 b^{2}+2$ \\
\hline
\end{tabular}

Proof. By Proposition 2.4(a) we know that $c=1$. Inserting this number into Fong's decomposition matrix, Lemma 3.1 follows from Theorem 48.8 of Dornhoff [4].

COROLlaRY 3.2. $\operatorname{Ext}_{F R(q)}^{1}\left(\varphi_{3}, \varphi_{3}\right)=0$.

LEMMA 3.3. $\operatorname{dim}_{F} \operatorname{Ext}_{F R(q)}^{1}\left(\varphi_{2}, \varphi_{2}\right)<1$. 
Proof. By Propositionn 2.4(a), $\varphi_{2 \mid N}=\frac{3^{*}}{3} \oplus$ projectives. Hence

$$
\operatorname{dim}_{F} \operatorname{Ext}_{F R(q)}^{1}\left(\varphi_{2}, \varphi_{2}\right)<\operatorname{dim}_{F} \operatorname{Ext}_{F N}^{1}\left(f\left(\varphi_{2}\right), f\left(\varphi_{2}\right)\right)
$$

by Lemma 1.1 of [12]. Now Proposition 2.3 of [12] asserts that $\Omega f\left(\varphi_{2}\right)$ has Loewy series

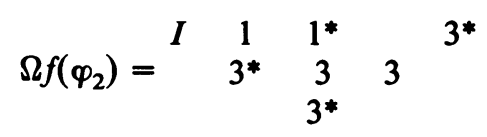

By Feit [6],

$$
\begin{aligned}
\operatorname{dim}_{F} \operatorname{Ext}_{F N}^{1}\left(f\left(\varphi_{2}\right), f\left(\varphi_{2}\right)\right) & =\operatorname{dim}_{F}\left(\Omega f\left(\varphi_{2}\right), f\left(\varphi_{2}\right)\right)_{N}^{1} \\
& <\operatorname{dim}_{F}\left(\Omega f\left(\varphi_{2}\right), f\left(\varphi_{2}\right)\right)=1,
\end{aligned}
$$

where the last equality follows from (*) and Proposition 2.3 of [12].

LEMMA 3.4. $\operatorname{dim}_{F} \operatorname{Ext}_{F R(q)}^{1}\left(\varphi_{i}, \varphi_{2}\right)=1$ for $i=4,5$.

Proof. By Fong's decomposition matrix of $B$,

$$
\operatorname{dim}_{F} \operatorname{Ext}_{F R(q)}^{1}\left(\varphi_{4}, \varphi_{2}\right)>1 \text {. }
$$

Let $\varphi_{4} \circ \varphi_{2}$ be any nonsplit extension of $\varphi_{2}$ by $\varphi_{4}$. As $\operatorname{dim}_{F}\left(\varphi_{4} \circ \varphi_{2}\right)$ is odd, it has vertex $E_{8}$.

By Lemma 2.2 and Proposition 2.4, $\varphi_{2}$ and $\varphi_{4}$ have generalized Green correspondents $f^{*}\left(\varphi_{2}\right)$ and $f^{*}\left(\varphi_{4}\right)$ in $C$. Furthermore,

$$
\left(\varphi_{4} \circ \varphi_{2}\right)_{\mid C}=f^{*}\left(\varphi_{4}\right) \circ f^{*}\left(\varphi_{2}\right) \oplus E_{2} \text {-projectives. }
$$

By the definition of relative projectivity $\left(\left[4\right.\right.$, p. 322]), also $Y=f^{*}\left(\varphi_{4}\right) \circ f^{*}\left(\varphi_{2}\right)$ is a nonsplit indecomposable extension with vertex $\operatorname{vx}(Y)={ }_{c} E_{8}$. Since $f^{*}\left(\varphi_{2}\right)_{\mid\langle u\rangle}$ is projective,

$$
\operatorname{ann}_{f^{\prime \prime}\left(\varphi_{2}\right)}(1-u) \cong I \oplus \chi^{\prime} \oplus \chi^{\prime \prime}<Y(1-u)
$$

As $Y$ has seven composition factors, we obtain

$$
Y(1-u)=I \oplus \chi^{\prime} \oplus \chi^{\prime \prime} \text { and } \operatorname{ann}_{Y}(1-u) / Y(1-u) \simeq \chi^{\prime \prime}
$$

Hence $Y / Y(1-u)$ is a semisimple $F C$-module. As $Y(1-u)<f^{*}\left(\varphi_{2}\right)$, this implies that $Y$ has Loewy length $j(Y)=2$. Since $Y$ is indecomposable, Lemma 1.6 asserts that $\operatorname{ann}_{Y}(1-u)$ is not a semisimple $F C^{\prime}$-module. Hence $Y_{\mid C^{\prime}}$ is indecomposable, because $f^{*}\left(\varphi_{2}\right)_{\mid C^{\prime}}$ is indecomposable. Therefore $f^{*}\left(\varphi_{2}\right)_{\mid C^{\prime}}$ has only one nonsplit extension by $\chi^{\prime \prime}$ as Lemma 1.1(a) shows. This completes the proof.

LEMMA 3.5. $\operatorname{Ext}_{F R(q)}^{1}\left(\varphi_{i}, \varphi_{i}\right)=0$ for $i=4,5$.

Proof. Suppose $\varphi_{4} \circ \varphi_{4}$ is a nonsplit extension. By Proposition 2.4(b),

$$
\varphi_{4 \mid C} e_{0}=\chi^{\prime \prime} \oplus \text { projectives. }
$$

Therefore by the definition of relative projectivity,

$$
\left(\varphi_{4} \circ \varphi_{4}\right)_{\mid} e_{0}=\chi^{\prime \prime} \circ \chi^{\prime \prime} \oplus \text { projectives, }
$$

where $\chi^{\prime \prime} \circ \chi^{\prime \prime}$ is a nonsplit extension. Hence $\chi^{\prime \prime} \circ \chi^{\prime \prime}$ has vertex $E_{4}$ by Lemma 1.1 and Green's theorem. Moreover, (*) and Lemma 1.1 imply 


$$
\left(\varphi_{4} \circ \varphi_{4}\right)_{\mid C^{\prime}} e_{0}=\chi^{\prime \prime} \oplus \chi^{\prime \prime} \oplus \text { projectives. }
$$

Since $\varphi_{4} \circ \varphi_{4}$ is $C^{\prime}$-projective, relative projectivity now asserts that $\varphi_{4} \circ \varphi_{4}$ is a split extension. By duality the other part of the lemma follows.

LEMMA 3.6. $\operatorname{Ext}_{F R(q)}^{1}\left(\varphi_{i}, \varphi_{j}\right)=0, i, j \in\{4,5\}$ and $i \neq j$.

Proof. Suppose $\varphi_{4} \circ \varphi_{5}$ is a nonsplit extension. As in the proof of the previous lemma, this implies

$$
\left(\varphi_{4} \circ \varphi_{5}\right)_{\mid C^{\prime}} e_{0}=\chi^{\prime \prime} \circ \chi^{\prime} \oplus \text { projectives, }
$$

where $\chi^{\prime \prime} \circ \chi^{\prime}$ again is a nonsplit extension. Moreover, $u$ lies in the kernel of $\chi^{\prime \prime} \circ \chi^{\prime}$. Thus

$$
\left(\chi^{\prime \prime} \circ \chi^{\prime}\right)_{\mid K}=\left(\chi^{\prime \prime} \circ \chi^{\prime}\right)_{\mid x_{4}}
$$

considered as a $K$-module by the trivial action of $u$. From Lemma 1.1(c) follows

$$
\begin{aligned}
\left(\varphi_{4} \circ \varphi_{5}\right)_{\mid K} & =\left(\chi^{\prime} \circ \chi^{\prime \prime}\right)_{\mid K} \oplus E_{2} \text {-projectives } \\
& =1^{*} \circ 1 \oplus E_{2} \text {-projectives. }
\end{aligned}
$$

As $\varphi_{4} \circ \varphi_{5}$ has vertex $E_{8}$, its Green correspondent $f\left(\varphi_{4} \circ \varphi_{5}\right)$ is a component of $\left(1^{*} \circ 1\right)^{N}$. Since $N$ is 2-closed, $f\left(\varphi_{4} \circ \varphi_{5}\right)$ has Loewy length 2 by (*). By Lemma 2.6 of [12] every $E_{2}$-projective $F K$-module has Loewy length at least 3 . Hence $f\left(\varphi_{4} \circ \varphi_{5}\right)_{\mid K}=1^{*} \circ 1$ by (*). Thus $\operatorname{dim}_{F} f\left(\varphi_{4} \circ \varphi_{5}\right)=2$, a contradiction to Proposition 2.3 of [12].

LEMMA 3.7. For each $\varphi \in\left\{I, \varphi_{4}, \varphi_{5}\right\}$ there exists at most one uniserial $F R(q)$-mod$\varphi_{2}$ ule $\varphi$.

$\varphi_{2}$ $\underset{\varphi_{2}}{\varphi_{2}}$. By Lemma 2.1(c), their largest isomorphic submodule is $\underset{\varphi_{2}}{I}$. Amalgamating it,

Proof. Suppose there are two nonisomorphic $F R(q)$-modules with Loewy series $\varphi_{2}$ we obtain an $F R(q)$-module with socle series

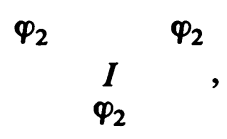

which is a contradiction to Lemma 2.1(c).

Since $\varphi_{2}$ is self-dual, Lemma 3.4 asserts

$$
\operatorname{dim}_{F} \operatorname{Ext}_{F R(q)}^{1}\left(\varphi_{2}, \varphi_{4}\right)=1=\operatorname{dim}_{F} \operatorname{Ext}_{F R(q)}^{1}\left(\varphi_{4}, \varphi_{2}\right) \text {. }
$$

Therefore the above argument shows that there exists at most one uniserial $F R(q)$-module

$$
\begin{aligned}
& \varphi_{2} \\
& \varphi_{4} \\
& \varphi_{2}
\end{aligned}
$$

As $\varphi_{4}$ and $\varphi_{5}$ are algebraic conjugate, the proof is complete. 
After all these preparations we now can compute the remaining parameters $a$ and $b$ of Fong's decomposition matrix of the principal 2-block $B$ of the groups $R(q)$ of Ree type.

Proposition 3.8. (a) Let $V$ be the indecomposable FR(q)-module of Lemma 2.3. Then it has socle series

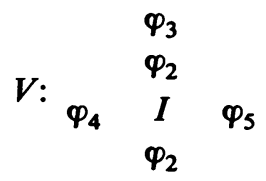

(b) The permutation module $U$ of Lemma 2.1 has socle series

$\begin{array}{ccc} & I & \\ & \varphi_{2} & \\ & \varphi_{4} & \varphi_{3} \\ & \varphi_{2} & \\ & I & \end{array}$

(c) $a=2$ and $b=1$.

Proof. Let $U$ be the permutation module of Lemma 2.1 and let $W$ be the one of Lemma 2.3. By the latter,

$$
W \cong I \oplus V \oplus V^{*}
$$

where soc $V=\varphi_{2}$ and soc $V^{*}=\varphi_{3}$. Furthermore, it follows from Lemma 2.3(a) and (e) that modulo $\pi R$, any $R$-form of $\xi_{3}$ has the same composition factors (including multiplicities) as $V$. Hence $I$ has multiplicity 1 in $V$ by Fong's decomposition matrix.

Since

$$
\begin{gathered}
I \\
\varphi_{2} \\
I
\end{gathered}
$$

is not projective by Lemma 2.1, it follows from the proof of Proposition 2.5(a) that $\left.\underset{\varphi_{2}}{I}\right|_{c} e_{0}$ has Loewy length 2 . Therefore Lemma 2.2(b) implies that $I$ occurs twice in the head of $\frac{I}{\varphi_{2}} l_{c}$. Hence

$$
\operatorname{dim}_{F}\left(\underset{\varphi_{2}}{I}, W\right)_{R(q)}=\operatorname{dim}_{F}\left(\left.\begin{array}{c}
I \\
\varphi_{2}
\end{array}\right|_{C}, I\right)_{C}=2
$$

Therefore $\frac{I}{\varphi_{2}}$ is a submodule of $V$, and $\operatorname{dim}_{F}(U, V)_{R(q)}=1$ by the multiplicity of $I$ in $V$.

Now Lemma $2.3(\mathrm{~d})$ asserts that $\operatorname{dim}_{F}(U, V)_{R(q)}=1$. Hence there exists a submodule $T$ of $V^{*}$ with soc $T=\varphi_{3}$ and head $(T)=I$.

Since $\operatorname{dim}_{F}\left(V^{*}, V\right)=1$ by Lemma $2.3(\mathrm{c})$ and (e), there is no submodule of $V$ with socle series $\varphi_{2}$, because by Lemma 3.3 , there exists at most one nonsplit extension of $\varphi_{2}$ by itself. Lemma 3.1 asserts that $\varphi_{3}$ occurs only once as a composition factor of $V$, namely as the head of $V$. Furthermore, the Loewy length $j(V)>3$, because $a>2$ by Lemma 2.1. Since $V$ is algebraically invariant, it 
follows that either

$$
\begin{array}{ccccc}
\varphi_{2} & & \varphi_{4} \\
I & \text { or } & \text { and } \varphi_{5} \\
\varphi_{2} & & & &
\end{array}
$$

exist as submodules of $V$. By Lemma 3.7 there is at most one uniserial $F R(q)$-module

$$
\underset{\varphi_{2}}{\varphi_{2}} .
$$

If it were a submodule of $V$, then Lemma 2.3 would imply that $2<$ $\operatorname{dim}_{F}\left(V^{*}, V\right)_{R(q)}=1$, a contradiction. Hence the second socle of $V$ is $S_{2}(V) / \varphi_{2} \simeq$ $\varphi_{4} \oplus I \oplus \varphi_{5}$ by Lemma 3.4.

By Lemma 2.1(c), $\operatorname{Ext}_{F R(q)}^{1}\left(\varphi_{i}, I\right)=0$ for $i=4,5$. Therefore Lemma 3.6 implies that only $\varphi_{2}$ can occur as a composition factor of the third socle $S_{3}(V)$ of $V$.

If the multiplicity of $\varphi_{2}$ in the third socle of $V$ is not 1 , then $V$ has a submodule $Y$ with socle series

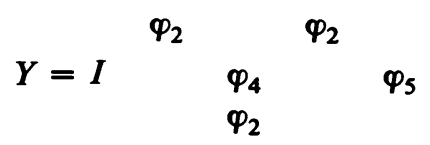

We claim that one of the $F R(q)$-modules

$$
\begin{array}{cccc}
\varphi_{2} & \varphi_{2} & & \varphi_{2} \\
I, & \varphi_{4} & \text { and } & \varphi_{5} \\
\varphi_{2} & \varphi_{2} & & \varphi_{2}
\end{array}
$$

is a submodule of $Y$.

By Lemma 1.6, the $F R(q)$-module $Y / \varphi_{2}$ is not indecomposable. As $\operatorname{soc}\left(Y / \varphi_{2}\right)=$ $I \oplus \varphi_{4} \oplus \varphi_{5}$, one of the direct summands is uniserial with $\varphi_{2}$ in its head. This proves our claim.

Now Lemma 3.7 and the claim yield $2<\operatorname{dim}_{F}\left(V^{*}, V\right)_{R(q)}=1$, a contradiction. Therefore $S_{3}(V) / S_{2}(V) \cong \varphi_{2}$.

As $\operatorname{dim}_{F}(V, U)_{R(q)}=1$ it now follows that there is a short exact sequence

$$
0 \rightarrow^{\varphi_{4}} \varphi_{2} \quad \varphi_{5} \rightarrow V \rightarrow T^{*} \rightarrow 0 .
$$

Since $\varphi_{2}$ occurs $a$ times in $V$, it has multiplicity $a-1$ in $T^{*}$.

Now $T^{*}$ is isomorphic to a submodule of $U$, and $\varphi_{3}$ is the head of $T^{*}$. Lemmas 2.1 and 3.1 assert that $\varphi_{3}$ occurs only once as a composition factor of $U$. Since $\varphi_{2}$ and $U$ are self-dual $F R(q)$-modules, and since $T$ is a factor module of $U$ with $\operatorname{soc}(T)=\varphi_{3}$, we obtain

$$
2(a-1)<a,
$$

because $\varphi_{2}$ has multiplicity $a$ in $U$ by Lemmas 2.1 and 3.1. Hence $a<2$. Therefore $a=2$. 
Applying now Lemmas 2.1, 3.4, 3.5 and 3.6, it follows that $U$ has one of the following socle series

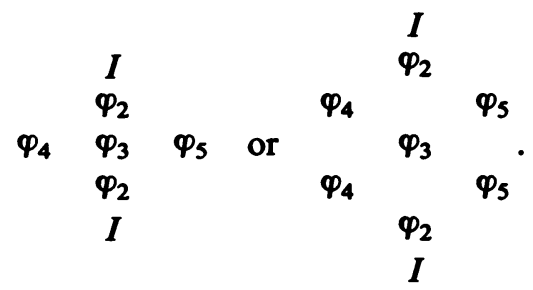

If the second possibility were true, then $T$ would have socle series

$$
T=\varphi_{4} \stackrel{\varphi_{2}}{ }{ } \varphi_{5} .
$$

As $T<V^{*}$, it follows that

$$
V=\begin{array}{ccc}
\varphi_{4} & \varphi_{3} & \\
& \varphi_{2} & \\
\varphi_{4} & I & \varphi_{5}
\end{array}
$$

In particular, $b=2$, and Lemma 2.1 implies that $\boldsymbol{P}_{\boldsymbol{I}}$ has socle series

$$
\begin{array}{ccccc} 
& & I & & \\
& & \varphi_{2} & & \\
& \varphi_{4} & I & \varphi_{5} & \\
& \varphi_{3} & & \varphi_{2} & \\
& \varphi_{5} & & \varphi_{4} & \varphi_{5} \\
& \varphi_{2} & & \varphi_{3} & \\
& \varphi_{4} & I & \varphi_{5} & \\
& & \varphi_{2} & & \\
& & I & &
\end{array}
$$

Therefore $P_{I}$ has one of the following factor modules

$$
X_{1}=\begin{gathered}
I \\
\varphi_{2} \\
I \\
\varphi_{2}
\end{gathered} \text { or } X_{2}=\begin{array}{ccc}
I & \\
\varphi_{4} & & \\
& I & \varphi_{5}
\end{array} \varphi^{\circ}
$$

In any case there is a self-dual indecomposable $F R(q)$-module $X$ with $\operatorname{soc}(X)=\varphi_{2}$ $=\operatorname{head}(X)$ such that there is a nonsplit short exact sequence

$$
0 \rightarrow I \rightarrow X_{i}^{*} \rightarrow X \rightarrow 0
$$

for some $i \in\{1,2\}$. Therefore by [14] we have

$$
0 \rightarrow \operatorname{Ext}_{F R(q)}^{1}\left(I, X_{i}^{*}\right) \rightarrow \operatorname{Ext}_{F R(q)}^{1}(I, X) \rightarrow 0,
$$

because $\operatorname{Ext}_{F R(q)}^{1}(I, I)=0=\operatorname{Ext}_{F R(q)}^{2}(I, I)$. By the structure of $P_{I}$,

$$
\operatorname{dim}_{F} \operatorname{Ext}_{F R(q)}^{1}(I, X)=1 \text {. }
$$


Hence either

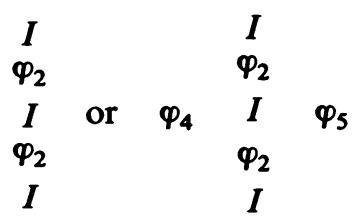

exists. However, $I$ is not a composition factor of the fifth socle of $P_{I}$, a contradiction. Thus $U$ has socle series

$$
\begin{array}{ccc} 
& I & \\
& \varphi_{4} & \\
& \varphi_{3} & \varphi_{5} \\
& \varphi_{2} & \\
& I &
\end{array}
$$

Hence $b=1$, and $V$ has a socle series as asserted in (a). This completes the proof of Proposition 3.8.

Combining Proposition 2.4 with Proposition 3.8, we obtain

TheOREM 3.9. (a) The principal 2-block $B$ of a group $R(q)$ of Ree type has decomposition matrix

\begin{tabular}{c|c|c|c|c|c|c} 
character & $I$ & $\varphi_{2}$ & $\varphi_{3}$ & $\varphi_{4}$ & $\varphi_{5}$ & degree \\
\hline$I$ & 1 & 0 & 0 & 0 & 0 & 1 \\
\hline$\xi_{2}$ & 1 & 1 & 0 & 0 & 0 & $q^{2}-q+1$ \\
\hline$\xi_{3}$ & 1 & 2 & 1 & 1 & 1 & $q^{3}$ \\
\hline$\xi_{4}$ & 1 & 1 & 1 & 1 & 1 & $q\left(q^{2}-q+1\right)$ \\
\hline$\xi_{5}$ & 0 & 1 & 0 & 1 & 0 & $\frac{1}{2}(q-1) m(q+1+3 m)$ \\
\hline$\xi_{7}$ & 0 & 1 & 0 & 0 & 1 & $\frac{1}{2}(q-1) m(q+1+3 m)$ \\
\hline$\xi_{6}$ & 0 & 0 & 0 & 1 & 0 & $\frac{1}{2}(q-1) m(q+1-3 m)$ \\
\hline$\xi_{8}$ & 0 & 0 & 0 & 0 & 1 & $\frac{1}{2}(q-1) m(q+1-3 m)$ \\
\hline & \multicolumn{1}{|c}{}
\end{tabular}

(b) B has Cartan matrix

\begin{tabular}{c|c|c|c|c|c} 
& $I$ & $\varphi_{2}$ & $\varphi_{3}$ & $\varphi_{4}$ & $\varphi_{5}$ \\
\hline$I$ & 4 & 4 & 2 & 2 & 2 \\
\hline$\varphi_{2}$ & 4 & 8 & 3 & 4 & 4 \\
\hline$\varphi_{3}$ & 2 & 3 & 2 & 2 & 2 \\
\hline$\varphi_{4}$ & 2 & 4 & 2 & 4 & 2 \\
\hline$\varphi_{5}$ & 2 & 4 & 2 & 2 & 4 \\
\hline
\end{tabular}


4. Socle series of the indecomposable projective $B$-modules. In this section we determine the structure of the indecomposable projective $F R(q)$-modules $P_{n}, n \in$ $\{I, 2, \ldots, 5\}$ belonging to the principal 2-block $B$ of a group $R(q)$ of Ree type.

THeOREM 4.1. Let $B$ be the principal 2-block of a group $R(q)$ of Ree type. Then the nonisomorphic indecomposable projective $F R(q)$-modules $P_{1}, P_{2}, \ldots, P_{5}$ of $B$ have socle and Loewy series as stated in the introduction.

Proof. By Lemma 2.1 and Proposition 3.8(b), the indecomposable projective $B$-module $P_{I}$ has the asserted socle and Loewy series.

The structure of $P_{3}$ follows immediately from Proposition 3.8(a), Theorem 3.9 and the self-duality of $P_{3}$. Hence the socle series of $P_{I} / I$ and $P_{3} / \varphi_{3}$ are

$$
\begin{aligned}
& I \\
& \varphi_{2}
\end{aligned}
$$

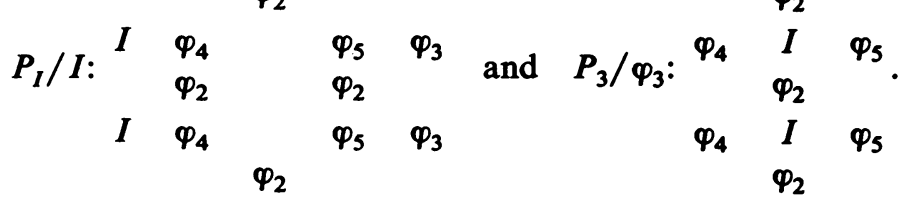

Furthermore, in both modules the socle and Loewy series coincide. By Lemma 2.3, $P_{2}$ contains a submodule $Y$ with socle and Loewy series

$$
Y=\varphi_{4} \begin{array}{ccc}
\varphi_{2} & \\
& \varphi_{2} & \varphi_{5}
\end{array}
$$

which is not self-dual.

Let $X=P_{I} / I$. Then Lemma 1.6 asserts that

$$
S_{3}(X) / S_{1}(X) \simeq\left(\begin{array}{lll} 
& \varphi_{2} & \\
I & \varphi_{4} & \varphi_{5}
\end{array}\right) \oplus\left(\begin{array}{l}
\varphi_{2} \\
\varphi_{3}
\end{array}\right)
$$

Hence

$$
\begin{aligned}
& \varphi_{2} \\
& \varphi_{3} \\
& \varphi_{2}
\end{aligned}
$$

exists. Thus $Y \approx Y^{*}$ implies now that $S_{3}\left(P_{2}\right) / S_{2}\left(P_{2}\right)>\varphi_{2} \oplus \varphi_{2} \oplus \varphi_{2}$.

Since $P_{2}$ is self-dual, and since $\varphi_{2}$ has multiplicity 8 in $P_{2}$, by Theorem 3.9 and Lemma 3.1, it follows that $\operatorname{Ext}_{F R(q)}^{1}\left(\varphi_{2}, \varphi_{2}\right)=0$, and

$$
\begin{array}{lllllll} 
& S_{3}\left(P_{2}\right)=I & \varphi_{2} & & \varphi_{2} & & \varphi_{2} \\
& & \varphi_{4} & & \varphi_{5} & & \varphi_{3} .
\end{array}
$$

Using the self-duality of $P_{2}$, the structure of $P_{I} / I$ and $P_{3} / \varphi_{3}$, it is now easy to see that $P_{2}$ has the asserted socle and Loewy series.

As $P_{4} \simeq\left(P_{5}\right)^{*}$, it suffices to determine the structure of $P_{4}$. The socle series of $P_{3}$ implies

$$
\operatorname{Ext}_{F R(q)}^{1}\left(\varphi_{4}, \varphi_{3}\right)=0=\operatorname{Ext}_{F R(q)}^{1}\left(\varphi_{3}, \varphi_{4}\right)
$$


Furthermore, $\operatorname{Ext}_{F R(q)}^{1}\left(I, \varphi_{3}\right)=0$. Therefore Corollary 3.2 and Lemma 3.4 assert that $S_{2}\left(P_{4}\right) / \varphi_{4}=\varphi_{2}$.

Considering now the homomorphisms from $P_{3}$ into $P_{4}$ we find two submodules of $\boldsymbol{P}_{4}$ with socle series

$$
\varphi_{4} \begin{array}{ccccc} 
& \varphi_{3} & & & \\
& \varphi_{2} & & & \varphi_{3} \\
& I & \varphi_{5} & \text { and } & \varphi_{2} \\
& \varphi_{2} & & & \varphi_{4} \\
& \varphi_{4} & & &
\end{array}
$$

Hence $S_{3}\left(P_{4}\right) / S_{2}\left(P_{4}\right)=\varphi_{4} \oplus I \oplus \varphi_{5} \oplus \varphi_{3}$ by the socle series of $P_{2}$. By Lemma 3.1 and Theorem 3.9, $\varphi_{3}$ has multiplicity 2 in $P_{4}$. Therefore the existence of the two above submodules of $P_{4}$ implies that $P_{4}$ has Loewy length $j\left(P_{4}\right)=7$. Since $\varphi_{2}$ is the second socle of $P_{I}, P_{4}$ and $P_{5}$, it follows from Lemma 3.1 and Theorem 3.9 that $S_{4}\left(P_{4}\right) / S_{3}\left(P_{4}\right)=\varphi_{2} \oplus \varphi_{2}$. Using the duality between $P_{4}$ and $P_{5}$, it is now easy to complete the proof of Theorem 4.1.

5. The Green correspondents of all simple $B$-modules. Let $f$ be the Green correspondence between $R(q)$ and $N=N_{R(q)}\left(E_{8}\right)$ with respect to a Sylow 2-subgroup $E_{8}$ of a group $R(q)$ of Ree type. By Proposition 2.5 it suffices to determine the structure of $f\left(\varphi_{3}\right)$. This is done in this section.

LEMMA 5.1. $\varphi_{3 \mid L}$ is a projective FL-module.

Proof. Let $X$ be an $R$-form of $\xi_{4}$ such that $\bar{X}=X / X \pi$ has socle $\varphi_{2}$. Then Theorem 3.9 and Theorem 4.1 imply that $\bar{X}$ has socle series

$$
\bar{X}=\begin{array}{lllll}
I & \varphi_{3} & & \varphi_{4} & \varphi_{5}
\end{array}
$$

By Lemma 2.2, $\varphi_{2 \mid L}=\Lambda_{q}$ is projective. Lemma 1.3 asserts

$$
\varphi_{4 \mid L}=\frac{1}{2}(m-1) \Lambda_{q} \oplus \Lambda_{m / 2}, \quad \varphi_{s \mid L}=\frac{1}{2}(m-1) \Lambda_{q} \oplus \bar{\Lambda}_{m / 2} .
$$

Therefore there are two indecomposable $B$-modules $Y$ and $Y^{\prime}$ with $\operatorname{soc}(Y)=\varphi_{4}$ and $\operatorname{soc}\left(Y^{\prime}\right)=\varphi_{5}$ such that

$$
\left(\Lambda_{m / 2}\right)^{R(q)} e=Y \oplus \frac{1}{2}(m-1) P_{3}, \quad\left(\bar{\Lambda}_{m / 2}\right)^{R(q)} e=Y^{\prime} \oplus \frac{1}{2}(m-1) P_{3},
$$

and $E_{2}$ is a vertex of $Y$ and $Y^{\prime}$. By means of Nakayama's relations it follows that $\Lambda_{m / 2}$ and $\bar{\Lambda}_{m / 2}$ occur both $\frac{1}{2}(m-1)$ times in the socle of $\varphi_{3 \mid L}$. Hence,

$$
\begin{aligned}
\xi_{4 \mid L}= & I^{-}+\Lambda_{1}+(q-1) \Lambda_{q}+m\left(\Lambda_{m}+\bar{\Lambda}_{m}\right)+\frac{1}{2}(m+1) \Lambda_{m / 2} \\
& +\frac{1}{2}(m-1) \Lambda_{m / 2}^{-}+\frac{1}{2}(m+1) \bar{\Lambda}_{m / 2}+\frac{1}{2}(m-1) \bar{\Lambda}_{m / 2}^{-}
\end{aligned}
$$

implies

$$
\begin{aligned}
\bar{X}_{\mid L}= & I \oplus \Lambda_{m / 2} \oplus \bar{\Lambda}_{m / 2} \oplus \Lambda_{1} \oplus(q-1) \Lambda_{q} \oplus m\left(\Lambda_{m} \oplus \bar{\Lambda}_{m}\right) \\
& \oplus \frac{1}{2}(m-1) P_{m / 2} \oplus \frac{1}{2}(m-1) \bar{P}_{m / 2},
\end{aligned}
$$


where $P_{m / 2}$ and $\bar{P}_{m / 2}$ denote the projective covers of $\Lambda_{m / 2}$ and $\bar{\Lambda}_{m / 2}$ respectively. On the other side, (*) implies

$$
\bar{X}_{\mid L}=I \oplus \Lambda_{m / 2} \oplus \bar{\Lambda}_{m / 2} \oplus \varphi_{3 \mid L} \oplus m \Lambda_{q}
$$

Therefore $\varphi_{3 \mid L}$ is a projective $F L$-module by the character table of $L$ and the Krull-Remak-Schmidt theorem.

Proposition 5.2. (a) The Green correspondent $f\left(\varphi_{3}\right)$ of the simple $F R(q)$-module $\varphi_{3}$ in FN has socle and Loewy series

$$
f\left(\varphi_{3}\right)=3 \quad \begin{aligned}
& 3 \\
& 3^{*}
\end{aligned} \quad 3^{*}
$$

(b) $\varphi_{3 \mid N}=f\left(\varphi_{3}\right) \oplus$ projectives.

(c) $\varphi_{3}$ has a periodic projective resolution, and $\Omega^{7}\left(\varphi_{3}\right) \simeq \varphi_{3}$.

Proof. Let $e_{0}$ be the block idempotent of the principal 2-block $b$ of $C$, and let $Y=\left(\varphi_{3 \mid C}\right) e_{0}$. By Lemma 1.2, $Y$ has composition factors

$$
2(q-m) I+[3(q-m)-2] \chi^{\prime}+[3(q-m)-2] \chi^{\prime \prime}
$$

Let $X=\operatorname{ann}_{Y}(1-u)$. Then $X=Y(1-u)$ by Lemma 5.1, because all involutions of $R(q)$ are conjugate. Furthermore, $X$ is a self-dual, algebraically invariant $F C^{\prime}$-module.

By Lemma 2.3(e), I has multiplicity 1 in $\operatorname{soc}(X)=\operatorname{soc}(Y)$. Let $X_{I}$ be the indecomposable component of $X$ with $I$ in the socle. Then $X_{I}$ has one of the following socle series

$$
I,\left(\begin{array}{lll}
\chi^{\prime} & & \chi^{\prime \prime} \\
& I &
\end{array}\right),\left(\begin{array}{lllll} 
& \chi^{\prime} & & \chi^{\prime \prime} & \\
I & & \chi^{\prime} & & \chi^{\prime \prime}
\end{array}\right),\left(\begin{array}{lll}
I & \chi^{\prime} & \chi^{\prime \prime} \\
I & \chi^{\prime} & \chi^{\prime \prime}
\end{array}\right), \quad P_{I}^{\prime} .
$$

Moreover, any other nonprojective component of $X$ belongs to $\left\{x^{\prime}, \chi^{\prime \prime}, \begin{array}{l}\chi^{\prime}, \\ \chi^{\prime \prime}, \\ \chi^{\prime \prime}\end{array}\right\}$ by Donovan-Freislich [3]. Since $P_{\chi^{\prime}}^{\prime}$ and $P_{\chi^{\prime \prime}}^{\prime}$ occur with the same multiplicity as a direct summand of $X$, and since the even number $(q-m)$ is the multiplicity of $I$ in $X$, Lemma 1.1 implies that $X_{I} \neq I$. Let $X_{1}=X_{I}$ if $X_{I}$ is self-dual, and let $X_{1}=X_{I} \oplus X_{I}^{*}$ otherwise. Then there are integers $r, s, t \in \mathbf{N}$ such that

$$
X=X_{1} \oplus t\left(\chi^{\prime} \oplus \chi^{\prime \prime}\right) \oplus r\left(\begin{array}{c}
\chi^{\prime} \\
\chi^{\prime \prime}
\end{array} \begin{array}{c}
\chi^{\prime \prime} \\
\chi^{\prime}
\end{array}\right)+s\left(P_{\chi^{\prime}}^{\prime} \oplus P_{\chi^{\prime \prime}}^{\prime}\right)
$$

If $x_{1}$ is the multiplicity of $\chi^{\prime}$ in $X_{1}$ and $x_{2}$ is the one of $I$, then (*) and (**) imply

$$
x_{1}+t+2 r+3 s=\frac{1}{2}[3(q-m)-2], \quad x_{2}+2 s=q-m .
$$

Hence $2 x_{1}<2 x_{1}+4 r+2 t=3 x_{2}-2$, which shows that

$$
X_{I} \neq\left(\begin{array}{lllll} 
& \chi^{\prime} & & \chi^{\prime \prime} & \\
I & & \chi^{\prime} & & \chi^{\prime \prime}
\end{array}\right) .
$$

If $X_{I}=P_{I}^{\prime}$, then $t=1$ and $r=0$. From (**) follows

$$
X=P_{I}^{\prime} \oplus \chi^{\prime} \oplus \chi^{\prime \prime} \oplus s\left(P_{\chi^{\prime}}^{\prime} \oplus P_{\chi^{\prime \prime}}^{\prime}\right) \text {. }
$$


As $\operatorname{soc}(Y)=\operatorname{soc}(X)$, we obtain

$$
Y=P_{I} \oplus \underset{\chi^{\prime}}{\chi^{\prime}} \oplus \underset{\chi^{\prime \prime}}{\chi^{\prime \prime}} \oplus s\left(P_{\chi^{\prime}} \oplus P_{\chi^{\prime \prime}}\right)
$$

Hence $Y$ is $E_{4}$-projective, which is a contradiction, because $\varphi_{3}$ has vertex $\operatorname{vx}\left(\varphi_{3}\right)=$ $E_{8}$ by Knörr's theorem [10]. Thus $X_{I} \neq P_{I^{\prime}}$, and in any of the remaining two cases (**) implies

$$
X=X_{1} \oplus s\left(P_{\chi^{\prime}}^{\prime} \oplus P_{\chi^{\prime \prime}}^{\prime}\right)
$$

where $s=\frac{1}{2}(q-m-2)$. Furthermore, $\operatorname{soc}\left(X_{1}\right)=I \oplus \chi^{\prime} \oplus \chi^{\prime \prime}$.

Let $T$ be the nonprojective part of $Y$. Then

$$
\begin{gathered}
\operatorname{soc}(T)=\operatorname{soc}\left(X_{1}\right)=I \oplus \chi^{\prime} \oplus \chi^{\prime \prime}=\operatorname{head}(T), \\
S_{2}(T) / S_{1}(T)=2 I \oplus 2 \chi^{\prime} \oplus 2 \chi^{\prime \prime},
\end{gathered}
$$

and $T$ has Loewy length $j(T)=3$. If $T$ were decomposable, then

$$
T \cap X=\chi^{\prime} \quad I \quad \chi^{\prime \prime} \oplus \chi^{\prime} \quad \chi^{\prime \prime} .
$$

Since $T(1-u)=T \cap X$ and $T$ have the same socle, it follows that $T=T_{1} \oplus T_{1}^{*}$ for some indecomposable $F C$-module, $T_{1}$ satisfying $T_{1} \cap X=\chi_{I}^{\prime} \quad \chi^{\prime \prime}$. Hence,

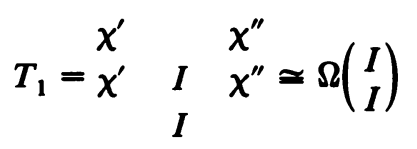

by Lemma 1.1. Therefore $T_{1}$ and hence $\varphi_{3}$ are $E_{4}$-projective. Again by Knörr's theorem [10], this is a contradiction. Thus $T$ is an indecomposable $F C$-module, and

$$
\varphi_{3 \mid C}=T \oplus \text { projective } F C \text {-modules }
$$

by Nagao's lemma [4, p. 353]. Furthermore,

$$
T \cap X=\operatorname{ann}_{T}(1-u)=T(1-u)=\begin{array}{lll}
I & \chi^{\prime} & \chi^{\prime \prime} \\
I & \chi^{\prime} & \chi^{\prime \prime}
\end{array}
$$

or

$$
\chi^{\prime} \quad x^{\chi^{\prime \prime}} \oplus_{\chi^{\prime}} \quad \chi^{\prime \prime}
$$

In particular, $T \cap X$ is an $F C^{\prime}$-module. By Lemma 1.1, its restriction to $K$ decomposes as follows.

$$
(T \cap X)_{\mid K}=\begin{array}{lll}
I & 1 & 1^{*} \\
I & 1 & 1^{*}
\end{array} \text { projective } F \mathfrak{U}_{4^{-m o d u l e s}}
$$

or

$$
(T \cap X)_{\mid K}=1 \quad I^{1^{*}} \bigoplus_{1} \quad I \quad 1^{*} \oplus \text { projective } F \mathfrak{A}_{4} \text {-modules. }
$$

As $T$ restricted to any involution is projective, the socles of $T_{\mid K}$ and $(T \cap X)_{\mid K}=$ $T_{\mid K}(1-u)$ coincide. Since there is a Green correspondence between $C$ and $K$ with respect to $E_{8}$, it follows that 


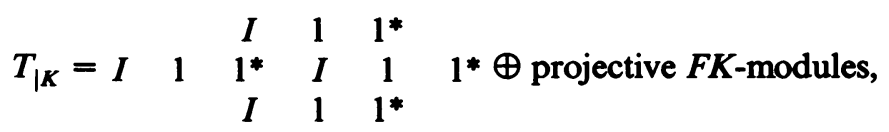

where the nonprojective part of $T_{\mid K}$ is indecomposable.

Now let $f\left(\varphi_{3}\right)$ be the Green correspondent of $\varphi_{3}$ in $N$. Since $N$ is 2-closed, $f\left(\varphi_{3}\right)_{\mid K}$ is indecomposable and coincides with the nonprojective part of $T_{\mid K}$. Therefore, $\operatorname{dim}_{F} f\left(\varphi_{3}\right)=12$. As $\varphi_{3}$ is self-dual and algebraically invariant, Proposition 2.3 of [12] implies that $f\left(\varphi_{3}\right)$ has composition factors $2 \cdot 3+2 \cdot 3$. Since $f\left(\varphi_{3}\right)_{\mid K}$ has Loewy length 3, also $f\left(\varphi_{3}\right)$ has Loewy length 3. Thus

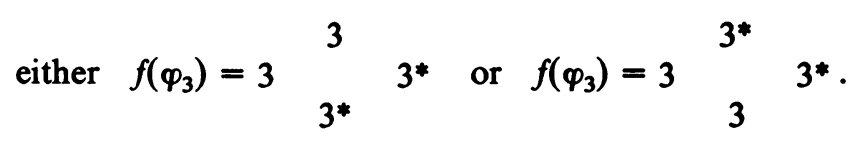

In either case, $(* * *)$ implies

$$
\varphi_{3 \mid N}=f\left(\varphi_{3}\right) \oplus \text { projective } F N \text {-modules, }
$$

which proves assertion (b).

By Theorem 4.1, $\operatorname{dim}_{F} \operatorname{Ext}_{F R(q)}^{1}\left(\varphi_{3}, \varphi_{2}\right)=1$. Hence,

$$
\operatorname{dim}_{F} \operatorname{Ext}_{F N}^{1}\left(f\left(\varphi_{3}\right), f\left(\varphi_{2}\right)\right)=\operatorname{dim}_{F}\left(\Omega\left(f\left(\varphi_{3}\right)\right), f\left(\varphi_{2}\right)\right)_{N}^{1} \neq 0
$$

by (b), Proposition 2.5(a) and Lemma 1.1 of [12]. If

$$
f\left(\varphi_{3}\right)=3_{3}^{3^{*}} 3^{*},
$$

then

$$
\Omega\left(f\left(\varphi_{3}\right)\right)=\begin{array}{lllll}
I & & 1 & 3^{*} & \\
& & 3 & 3^{*}
\end{array}
$$

and

$$
\operatorname{Hom}_{F N}\left(\Omega\left(f\left(\varphi_{3}\right)\right), f\left(\varphi_{2}\right)\right)=\left(\Omega\left(f\left(\varphi_{3}\right)\right), f\left(\varphi_{2}\right)\right)_{N}^{1}=0
$$

by Proposition 2.5(a). This contradiction proves assertion (a).

In order to prove (c) by Green [9, p. 151], it suffices to show that $\Omega^{7} f\left(\varphi_{3}\right) \simeq f\left(\varphi_{3}\right)$. From (a) and Proposition 2.3 of [12], it follows that

$$
0 \leftarrow f\left(\varphi_{3}\right) \leftarrow P_{3} \leftarrow P_{3^{*}} \leftarrow P_{3^{*}} \leftarrow\left(P_{1} \oplus P_{1} \oplus P_{1^{*}}\right) \leftarrow P_{3} \leftarrow P_{3} \leftarrow P_{3^{*}} \leftarrow f\left(\varphi_{3}\right) \leftarrow 0
$$

is a minimal projective resolution of $f\left(\varphi_{3}\right)$. Hence $\Omega^{7}\left(\varphi_{3}\right) \simeq \varphi_{3}$, which completes the proof of Proposition 5.2.

Combining the results of Propositions 2.5 and 5.2, we have shown

THEOREM 5.3. The simple $F R(q)$-modules belonging to the principal 2-block $B$ of $a$ group $R(q)$ of Ree type have Green correspondents in FN with the following socle and Loewy series:

(a) $f(I)=I, f\left(\varphi_{4}\right)=1^{*}$, and $f\left(\varphi_{5}\right)=1$,

(b) $f\left(\varphi_{2}\right)=\frac{3^{*}}{3}$,

(c) $f\left(\varphi_{3}\right)=3$ 
6. An application. By means of Theorem 4.1 and recent work of $J$. Alperin [1], K. Erdmann [5] and the authors [12] it is now easy to give a sharp upper bound for the Loewy lengths $j(P)$ of the indecomposable projective $F G$-modules $P$ belonging to the principal 2-block $B_{0}$ of an arbitrary finite group $G$ with an abelian Sylow 2-subgroup. Since the index of nilpotency of the Jacobson radical $J=J(F G)$ is preserved by field extensions, the following result holds for arbitrary 2-modular systems $(F, R, S)$ for $G$.

THEOREM 6.1. Every indecomposable projective FG-module $P$ of the principal 2-block $B_{0}$ of a finite group $G$ with an abelian Sylow 2-subgroup $D$ of order $|D|=2^{n}$ has Loewy length $j(P)<\max \left\{2 n+1,2^{n}\right\}$.

Proof. Let $O(G)$ be the maximal normal subgroup of $G$ with odd order. Then by J. Walter's theorem (see Gorenstein [8, p. 485]) $G$ contains a normal subgroup $T \geqslant O(G)$ with odd index $|G: T|$ such that $T / O(G)$ is a direct product of a 2-group and simple groups of the following types:

(a) $G \cong \operatorname{PSL}(2, q), q>3, q \equiv 3$ or $5 \bmod 8$.

(b) $G \cong \operatorname{PSL}\left(2,2^{a}\right), a>1$.

(c) $G \cong J_{1}$, the smallest Janko group of order $\left|J_{1}\right|=175,560$.

(d) $G \cong R(q)$, a simple group of Ree type.

Let $j(A)$ be the index of nilpotency of the Jacobson radical of an algebra $A$. Then for every indecomposable projective $B_{0}$-module $P$ we have $j(P)<j\left(B_{0}\right)=j$.

Since $B_{0}$ is the principal 2-block of $G$, the elements of $O(G)$ act trivially on $B_{0}$. Hence we may assume that $O(G)=1$. As $|G: T|$ is odd, Villamayor's theorem (see $[13$, p. 524]) asserts that $j(F G)=j(F T)$. Thus we may also assume that $G=T$.

If $G=G_{1} \times G_{2}$, then $F G=F G_{1} \otimes_{F} F G_{2}$. From Theorem 71.10 of CurtisReiner [2, p. 485], it follows easily that Jacobson radical

$$
J(F G)=J\left(F G_{1}\right) \otimes F G_{2}+F G_{1} \otimes J\left(F G_{2}\right) .
$$

Therefore $j\left(B_{0}(G)\right)=j\left(B_{0}\left(G_{1}\right)\right)+j\left(B_{0}\left(G_{2}\right)\right)-1$. Now suppose that the assertion of Theorem 6.1 holds in $G_{1}$ and $G_{2}$. Let $p^{d_{i}}$ be the order of a Sylow 2-subgroup $S_{i}$ of $G_{i}, i=1,2$. Then $S_{1} \times S_{2}$ is a Sylow 2-subgroup of $G=G_{1} \times G_{2}$ with order $p^{\left(d_{1}+d_{2}\right)}$. Hence

$$
\begin{aligned}
j\left(B_{0}(G)\right) & <\max \left\{2 d_{1}+1,2^{d_{1}}\right\}+\max \left\{2 d_{2}+1,2^{d_{2}}\right\}-1 \\
& <\max \left\{2\left(d_{1}+d_{2}\right)+1,2^{\left(d_{1}+d_{2}\right)}\right\} .
\end{aligned}
$$

Therefore we may assume that $G$ is either an abelian 2-group or one of the simple groups of Walter's list (a)-(d). If $G$ is abelian, then $j\left(B_{0}\right)<|D|$. In case (a), $j\left(B_{0}\right)<\max \left\{2 n+1,2^{n}\right\}$ (see K. Erdmann [5] $]^{4}$. In case (b), $j\left(B_{0}\right)=2 n+1$ by Theorem 4 of J. Alperin [1]. In case (c), $j\left(B_{0}\right)=7=2 n-1$ by Theorem 6.7 of [12]. Finally, Theorem 4.1 asserts that also $j\left(B_{0}\right)=7=2 n-1$, if $G$ is a group $R(q)$ of Ree type. Thus Theorem 6.1 holds.

${ }^{4}$ See Footnote 3. 


\section{REFERENCES}

1. J. L. Alperin, Projective modules for SL $\left(2,2^{n}\right)$, University of Chicago, 1978 (preprint).

2. C. W. Curtis and I. Reiner, Representation theory of finite groups and associative algebras, Wiley, New York, 1962. MR 26 \#2519.

3. P. W. Donovan and M. R. Freislich, Indecomposable representations in characteristic two of the simple groups of order not divisible by eight, Bull. Austral. Math. Soc. 15 (1976), 407-419. MR 55 \#3055.

4. L. Dornhoff, Group representation theory. Part B, Dekker, New York, 1972. MR 50 \# 458b.

5. K. Erdmann, Principal 2-blocks of groups with dihedral Sylow 2-subgroups, Comm. Algebra 5 (1977), 665-694. 1969.

6. W. Feit, Representations of finite groups. I, Lecture Notes, Yale University, New Haven, Conn.,

7. P. Fong, On the decompasition numbers of $J_{1}$ and $R(q)$, Sympos. Math. Rome 13 (1972), 415-422; Academic Press, London, 1974. MR 50 \# 10046.

8. D. Gorenstein, Finite groups, Harper and Row, New York, 1968. MR 38 \#229.

9. J. A. Green, Vorlesungen über modulare Darstellungen endlicher Gruppen, Vorlesungen Math. Inst. Universität Giessen, 1974. MR 50 \# 13235

10. R. Knörr, On the vertices of irreducible modules (to appear).

11. P. Landrock, On centralizers of p-elements in indecomposable modules (to appear).

12. P. Landrock and G. O. Michler, Block structure of the smallest Janko group, Math. Ann. 232 (1978), 205-238.

13. G. Michler, Blocks and centers of group algebras, Lecture Notes in Math., vol. 246, SpringerVerlag, Berlin and New York, 1972, pp. 429-563. MR 48 \# 11274.

14. L. Scott, Permutation modules and 1-cohomology, Arch. Math. 27 (1976), 362-368. MR 56 \#689

15. H. N. Ward, On Ree's series of simple groups, Trans. Amer. Math. Soc. 121 (1966), 62-89. MR 33 \#5752

Department of Mathematics, Aarhus Universitet, 8000 Aarhus, Denmark

Department of Mathematics, Universität Essen, 4300 Essen, Federal Republic of Germany 